\title{
Multi-step limit cycle generation for Rabbit's walking based on a nonlinear low dimensional predictive control scheme
}

\author{
Ahmed Chemori *, Mazen Alamir \\ Laboratoire d'Automatique de Grenoble, UMR 5528, BP46, Domaine Universitaire, 38400 Saint Martin d'Hères, France
}

Received 22 February 2005; accepted 7 December 2005

In this paper, a new nonlinear predictive control scheme is proposed for a five-link planar under-actuated biped walking robot. The basic feature in the proposed strategy is to use on-line optimization to update the tracked trajectories in the completely controlled variables (actuated coordinates) in order to enhance the behavior and the stability of the remaining indirectly controlled ones (unactuated coordinates). The stability issue is discussed using the Poincaré's section tool leading to a computable criterion that enables the stability of the overall scheme to be investigated as well as the computation of a candidate region of attraction. The whole framework is illustrated hrough simulation case-studies. To attest the efficiency of the proposed scheme, robustness against model uncertainties and ground irregularities are investigated by simulation studies.

16 (c) 2006 Elsevier Ltd. All rights reserved.

Keywords: Biped robots; Dynamic walking; Multi-step limit cycle; Nonlinear optimization; Nonlinear predictive control; Orbital stability

[29,2]. An excellent database of climbing and walking 33 robots built all over the world can be found in [2]. One 34 of the serious reasons for exploring the use of legged robots 35 is the mobility [23], there is a need for vehicles that can tra- 36 vel in difficult terrains, where existing wheeled vehicles can- 37 not go, since wheels excel on prepared surfaces such as rails 38 and roads, but they perform poorly on rough terrains. 39 Moreover, walking robots could co-exist with their 40 Alamir@inpg.fr (M. Alamir). 
creators without any costly modification to the environment created for humans.

In walking locomotion [23], two gaits could be underlined. Static walking which refers to a system which stays balanced by always keeping the center of mass (c.o.m.) of the system vertically projected over the polygon of support formed by feet. On the contrary, dynamic walking $[24,21,13,22]$ is not constrained in such a manner, therefore the c.o.m. may leave the support polygon for periods of time. Biped robots $[23,34,24]$ have high mobility that allows them to achieve dynamic walking, consequently high speeds could be reached due to the horizontal acceleration.

Currently, many research groups in the world are working on biped robots, either on optimization of leg and foot trajectory, stable walking control, or hardware design. The main thrust of current research on biped control includes many proposed control approaches, such as intuitive control [28], intelligent learning control [19], neural network control [18], passivity based control [33], sliding control [6], impedance control [25], optimal control [14], computed torque control [5], and tracking control [31,30].

From a control viewpoint, the major academic interest of bipeds comes from (1) their hybrid nature resulting from the unavoidable impacts [3] with the ground which can produce discontinuities (jumps) in the generalized velocities. (2) Another interesting point is under-actuation, indeed biped walking robots may be under-actuated (case of Rabbit) that is the robot has fewer number of actuators than the number of degrees of freedom. The way this underactuation is handled may be used to give a particularly clear insight into the set of solutions proposed so far within the academic control community.

1. One way to overcome the under-actuation related-difficulty is to define virtual controls [8]. Typically, reference trajectories are defined on the whole state including indirectly controlled sub-states. These trajectories are dependent on some parameter vector $p$ that can be either a virtual time, a remaining free polynomial coefficient or both. Generally, the second time-derivative of the parameter $p$ becomes a virtual additional control enabling under-actuation to be conceptually overcome. Clearly, technical details are to be investigated when this second derivative is monitored by the tracking requirements (virtual time needs to be monotonic, coefficient excursions have to be compatible with geometric constraints, etc.) The reference trajectories to be tracked may be computed using classical constrained optimal control tools. Several optimization criterions have been proposed $[31,26,30]$.

2. A second way to handle under-actuation is to use the concept of virtual constraints and the associated zerodynamics $[12,35,36]$. Namely, some regulated output is suitably defined that can be exactly tracked using the available control inputs. The constrained dynamics of the remaining sub-state on the zero-output manifold is then called the zero-dynamics [17]. This methodology is therefore based on the analytical study of the resulting zero-dynamics that corresponds to each particular choice of the regulated output. If the latter is taken in a parameterized closed-loop form, off-line optimization can then be used to enhance the asymptotic stability of the zero-dynamics $[12,35,36]$. A particular feature when dealing with the zero-dynamics associated to bipeds is their hybrid nature [36].

3. A third way to handle under-actuation in nonlinear dynamical systems is predictive control schemes $[4,20,11]$. Indeed, these schemes ensure stability by controlling the behavior of the whole state at some future time, say $N$-sampling times ahead. This naturally suppresses under-actuation since the number of control d.o.f. is $r \times N$ where $r$ is the number of actuators while the controlled state is still $n$-dimensional. Therefore, under-actuation generically disappears as soon as $N r \geqslant n$.

The work proposed in this paper might be viewed as a mixture of the last two categories. Namely a nonlinear predictive control scheme is proposed for the control of a fivelink 7 d.o.f. under-actuated biped robot while the stability of the resulting zero-dynamics is explicitly studied. Contrary to the approach adopted in [1], where a somehow black-box formulation is used to define the auxiliary open-loop optimization problem, our approach leads to low dimensional decision variables. This may be crucial in a real-time implementation context. In particular, it is shown that with a scalar such open-loop optimization problem, provably stable and quasi-cyclic motions can be generated.

The basic differences between the approach proposed in this paper and existing provably stable limit-cycle generation can be summarized as follows:

- The limit cycle so-obtained may include several steps. Namely, the robot configuration just after the impact is not necessarily the same as the one just after the preceding impact. This happens especially with very low dimensional (scalar) predictive control. By increasing the d.o.f. of the control parameterization, classical one-step limit cycles may be recovered, but the underlying predictive control may not be real-time implementable.

- The resulting closed-loop trajectories do not necessarily correspond to a periodic motion of the torso. The latter converges to a neighborhood of a stable limit cycle. This is a crucial point since it has been pointed out in [27,9] that such periodic motion is hard to achieve with at least polynomial trajectories of the actuated variables.

Sufficient conditions for the stability of the feedback scheme are derived together with a concrete computation procedure to compute the corresponding region of attraction related to the zero-dynamics of the closed-loop system. 
This paper is organized as follows. First, the biped robot prototype is described in Section 2. Then the proposed predictive control approach is presented in a rather general setting (Section 3). Computable sufficient conditions for stability are derived and implementation related topics are discussed in Section 4. Finally simulation results are given in Section 5, illustrating the potentiality of the proposed solution. These simulations include scenarios where a stable walk is obtained from the rest position as well as transitions between different desired mean walking speeds. Robustness against model parameters uncertainties and ground irregularities are also verified. The paper ends by some concluding remarks.

\section{The RABBIT prototype description}

The academic prototype RABBIT [10] is a biped walking robot with five links and seven d.o.f. (see Fig. 1), which results from the joint effort of several French laboratories (Mechanical engineering, Automatic control, and Robotics) working on a project on control of walking robots. ${ }^{1}$ By means of guidance device, RABBIT walks in a circular path (see Fig. 2) while looking like a planar biped. The counter-balance should be used to offset the weight of the lateral stabilization bar in the guidance device. More technical details about the testbed can be found in [10].

\subsection{Dynamic model}

Using Lagrange formulation [32], the mathematical model describing the biped moving in the sagittal plane is as follows:

$M(q) \ddot{q}+N(q, \dot{q}) \dot{q}+G(q)=S u+F_{\text {ext }}$

where $M(q) \in \mathbb{R}^{7 \times 7}$ is the inertia matrix, $N(q, \dot{q}) \in \mathbb{R}^{7 \times 7}$ contains the centrifugal and Coriolis forces terms, $G(q) \in \mathbb{R}^{7}$ is the vector of gravitational forces, $u=\left[\begin{array}{llll}u_{1} & u_{2} & u_{3} & u_{4}\end{array}\right]^{\mathrm{T}} \in \mathbb{R}^{4}$ is the vector of control inputs, $S$ is a torque distribution matrix, $q=\left[\begin{array}{lllllll}q_{31} & q_{41} & q_{32} & q_{42} & q_{1} & x & y\end{array}\right]^{\mathrm{T}} \in \mathbb{R}^{7}$ is the vector of generalized coordinates (see Fig. 3). Finally, $F_{\text {ext }}$ represents the external forces acting on the robot (contact forces with the ground). Following the proposed decomposition of the walking cycle proposed in [12], the walking cycle can be divided into two consecutive phases of motion (see Fig. 4). In the first one, the biped remains in contact with the ground through one foot (single support (SS) phase). The other one is the impact phase [3] that is often considered as instantaneous and characterized by a collision between the swing leg and the ground. Since the assumption that the robot is walking on horizontal surface without obstacles is made, the switching from one walking phase to another is closely related to the vertical position of the robot free leg tip. Let this position be denoted by $\sigma(q)$, the stance leg is denoted by $\left(q_{31}, q_{41}\right)$, and the swing leg by $\left(q_{32}, q_{42}\right)$, therefore

\footnotetext{
${ }^{1}$ For a detailed information, see http://robot-rabbit.lag.ensieg.inpg.fr/
}

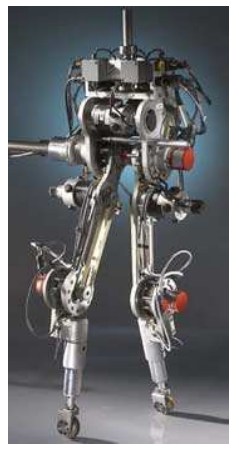

Fig. 1. RABBIT prototype testbed.

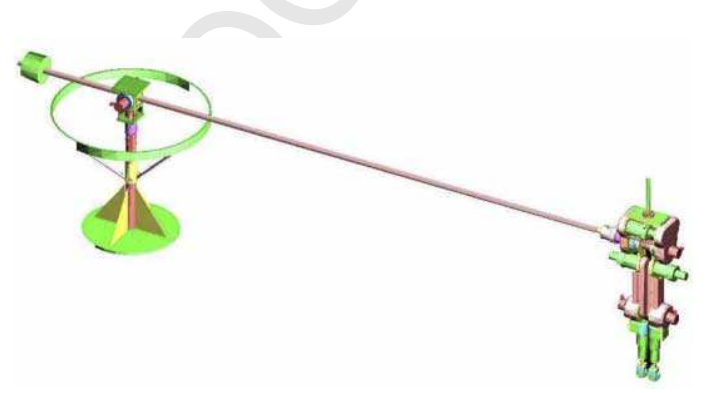

Fig. 2. The guidance device.

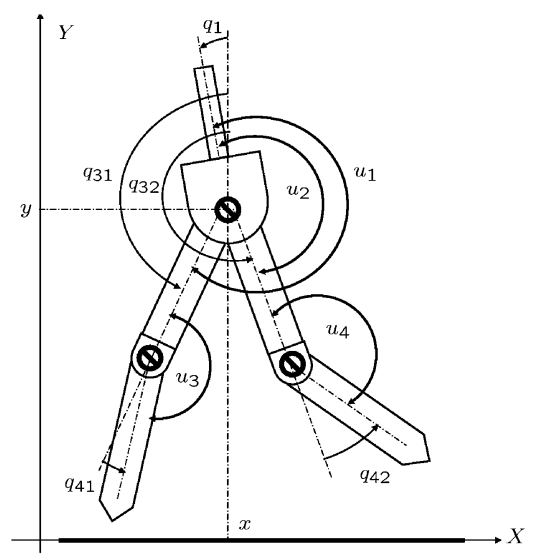

Fig. 3. Schematic view of RABBIT's mechanical structure.

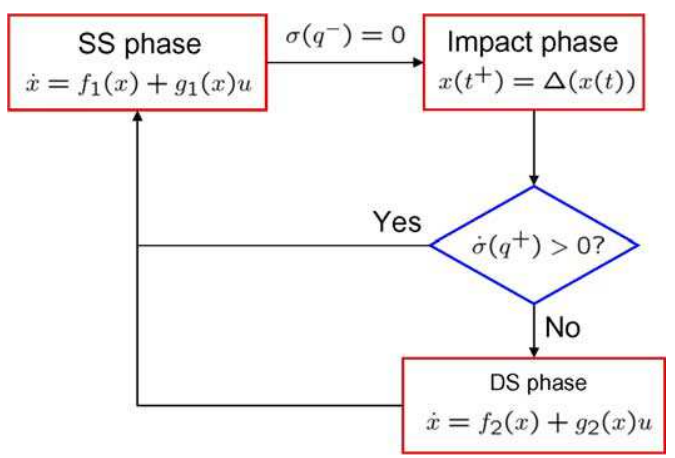

Fig. 4. The walking cycle decomposition. 


$$
\begin{aligned}
\sigma(q)= & l_{3}\left(\cos \left(q_{32}\right)-\cos \left(q_{31}\right)\right)+l_{4}\left(\cos \left(q_{32}+q_{42}\right)\right. \\
& \left.-\cos \left(q_{31}+q_{41}\right)\right)
\end{aligned}
$$

205 Indeed the impact between the swing leg and the ground ${ }^{2}$ 206 occurs when the foot hits the ground, which can be ex207 pressed as

$209 \sigma(q)=0 ; \quad \dot{\sigma}(q)<0$

210 and it characterizes the switch from the single support 211 phase to the impact phase (cf. Fig. 4). On the other hand, 212 the lift-off from ground occurs just after the impact [3] and 213 may be expressed as (after re-labelling the variables)

$215 \sigma\left(q^{+}\right)=0 ; \quad \dot{\sigma}\left(q^{+}\right)>0$

216 and it characterizes the switch from the impact phase to the 217 single support phase (cf. Fig. 4). Note that $q^{+}$denotes the 218 vector of the generalized coordinates just after the impact 219 (cf. Section 2.1.2). In the following sections, the dynamic 220 equations for these two phases are presented.

\section{2.1.1. The single support phase model}

222 In this phase only one foot is grounded, and the biped is 223 modelled by the following differential equation [32]:

$225 M(q) \ddot{q}+N(q, \dot{q}) \dot{q}+G(q)=S u+J_{1}^{\mathrm{T}}(q) \lambda$

226 where $J_{1}(q)$ represents the Jacobian matrix of the holo227 nomic contact constraints, and $\lambda$ the Lagrange multipliers 228 of contact forces. Assuming that $\left(q_{31}, q_{41}\right)$ is the stance 229 leg, the contact constraints may be expressed by

$232 y_{p_{1}}=\dot{y}_{p_{1}}=\ddot{y}_{p_{1}}=0 ; \quad x_{p_{1}}=\dot{x}_{p_{1}}=\ddot{x}_{p_{1}}=0$

233 where $\left(x_{p_{1}}, y_{p_{1}}\right)$ denotes the cartesian coordinates of the 2345 stance leg's foot, given by

$\left\{\begin{array}{l}y_{p_{1}}(q)=y+l_{3} \cos \left(q_{31}\right)+l_{4} \cos \left(q_{31}+q_{41}\right) \\ x_{p_{1}}(q)=x-l_{3} \sin \left(q_{31}\right)-l_{4} \sin \left(q_{31}+q_{41}\right)\end{array}\right.$

238 Using (5) and (4) one obtains

$240 J_{1}(q) \ddot{q}+\Pi_{2}(q, \dot{q})=0$

241 where $\Pi_{2}(q, \dot{q})$ is defined by

$\Pi_{2}(q, \dot{q}):=\left(\begin{array}{c}-l_{3} \dot{q}_{31}^{2} \cos \left(q_{31}\right)-l_{4}\left(\dot{q}_{31}+\dot{q}_{41}\right)^{2} \cos \left(q_{31}+q_{41}\right) \\ l_{3} \dot{q}_{31}^{2} \sin \left(q_{31}\right)+l_{4}\left(\dot{q}_{31}+\dot{q}_{41}\right)^{2} \sin \left(q_{31}+q_{41}\right)\end{array}\right)$

244 The constrained dynamic model in the single support phase is then given by

$$
\left\{\begin{array}{l}
M(q) \ddot{q}+N(q, \dot{q}) \dot{q}+G(q)=S u+J_{1}^{\mathrm{T}}(q) \lambda \\
J_{1}(q) \ddot{q}+\Pi_{2}(q, \dot{q})=0
\end{array}\right.
$$

249 In simulation of the walking robot during the single sup250 port phase, a reduced order dynamic model, computed 251 form (7), is used (cf. [7] and the references inside for more 252 details).

\subsubsection{The impact phase model}

According to [15], the impact between the swing leg and 254 the ground is considered as a rigid collision [3], it occurs when the swing leg hits the walking surface and it induces discontinuities (jumps) in the generalized velocities, ${ }^{3}$ our objective is then to derive the post-impact velocities in terms of pre-impact positions and velocities. During the impact we have

$M(q) \ddot{q}+N(q, \dot{q}) \dot{q}+G(q)=S u+\delta F_{\text {ext }}$

where $F_{\text {ext }}$ represents the external contact forces.

Under suitable assumptions (see e.g. [15]) on the impact 265 phenomenon, one can deduce the external acting forces by 266 integration of (8) over the impact duration, so one obtains

$M(q)\left(\dot{q}^{+}-\dot{q}^{-}\right)=F_{\text {ext }}=J_{2}^{\mathrm{T}}(q) \lambda$

where $\dot{q}^{+}$(respectively, $\dot{q}^{-}$) is the velocity just after (respectively before) impact, and $F_{\text {ext }}=\int_{t^{-}}^{t^{+}} \delta F_{\text {ext }} . J_{2}(q)$ is the Jacobian matrix of the cartesian coordinates of the swing leg foot, given by

$$
\left\{\begin{array}{l}
y_{p_{2}}(q)=y+l_{3} \cos \left(q_{32}\right)+l_{4} \cos \left(q_{32}+q_{42}\right) \\
x_{p_{2}}(q)=x-l_{3} \sin \left(q_{32}\right)-l_{4} \sin \left(q_{32}+q_{42}\right)
\end{array}\right.
$$

Eq. (9) involves seven constraints and nine unknowns $F_{\text {ext }}$ and $\dot{q}^{+}$. Two additional equations may be obtained from the condition that the impacted leg does not rebound nor slips at impact, that is

$y_{p_{2}}=\dot{y}_{p_{2}}^{+}=0 ; \quad x_{p_{2}}=\dot{x}_{p_{2}}^{+}=0$

which gives using the expressions of $x_{p_{2}}, y_{p_{2}}$

$J_{2}(q) \dot{q}^{+}=0$

The solution of (9)-(12) leads to

$$
\left\{\begin{array}{l}
\dot{q}^{+}=\left[I-M^{-1} J_{2}^{\mathrm{T}}\left(J_{2} M^{-1} J_{2}^{\mathrm{T}}\right)^{-1} J_{2}\right] \dot{q}^{-}=D(q) \dot{q}^{-} \\
\lambda=-\left[\left(J_{2} M^{-1} J_{2}^{\mathrm{T}}\right)^{-1} J_{2}\right] \dot{q}^{-}
\end{array}\right.
$$

On the other hand, the impact model must account for the re-labelling of the robot coordinates (i.e. the swing leg becomes the new stance leg and vice versa), this can be expressed by

$$
\left(\begin{array}{c}
q^{+} \\
\dot{q}^{+}
\end{array}\right)=R(q)\left(\begin{array}{c}
q^{-} \\
\dot{q}^{-}
\end{array}\right)
$$

To summarize, the global impact model that includes both the jumps in velocities and the permutation of coordinates and velocities shortly writes

$$
\left(\begin{array}{c}
q^{+} \\
\dot{q}^{+}
\end{array}\right)=\Delta(q)\left(\begin{array}{c}
q^{-} \\
\dot{q}^{-}
\end{array}\right)
$$

\footnotetext{
${ }^{2}$ The system looks like a kinematic chain [15].
}

\footnotetext{
${ }^{3}$ But the generalized positions still unaltered i.e. $q^{+}=q^{-}=q$.
} 


$$
\Delta(q)=\left(\begin{array}{cc}
R(q) & 0 \\
0 & R(q) D(q)
\end{array}\right) R=\left(\begin{array}{ccccccc}
0 & 0 & 1 & 0 & 0 & 0 & 0 \\
0 & 0 & 0 & 1 & 0 & 0 & 0 \\
1 & 0 & 0 & 0 & 0 & 0 & 0 \\
0 & 1 & 0 & 0 & 0 & 0 & 0 \\
0 & 0 & 0 & 0 & 1 & 0 & 0 \\
0 & 0 & 0 & 0 & 0 & 1 & 0 \\
0 & 0 & 0 & 0 & 0 & 0 & 1
\end{array}\right)
$$

\section{The key idea: a predictive control scheme}

Under single support assumption, the five independent degrees of freedom can be subdivided into two parts

$$
z_{1}:=q_{1} \in \mathbb{R} ; \quad z_{2}:=\left(\begin{array}{llll}
q_{31} & q_{41} & q_{32} & q_{42}
\end{array}\right)^{\mathrm{T}} \in \mathbb{R}^{4}
$$

where $z_{2}$ can be assumed to be completely controllable (provided that saturation constraints on actuators and contact conditions are fulfilled). In this section, the sequence of impact instants is denoted by $\left(t_{k}\right)_{k \in \mathbb{N}}$ with $t_{k}=k t_{f}$ where $t_{f}$ is the step duration.

Remark 1. Under nominal conditions, the step duration $t_{f}$ is fixed and it does not change from one step to another, nevertheless if the biped is required to change the walking speed, among others, a solution could be investigated to change this parameter, but for each speed and configuration corresponds a well specified value of $t_{f}$.

Let us choose some target configuration $z_{2}^{\mathrm{f}} \in \mathbb{R}^{4}$ (cf. Section 5.1) that is to be reached just before the impact instants $t_{k}$ that is $z_{2}\left(t_{k}^{-}\right)=z_{2}^{\mathrm{f}}$. This choice is fixed in all the forthcoming developments, in a way, $z_{2}^{\mathrm{f}}$ has to be considered as a design parameter. The way $z_{2}^{\mathrm{f}}$ may be parameterized is explained in Section 5.1.

Associated to this choice of $z_{2}^{\mathrm{f}}$, the following choice $\dot{z}_{2}^{\mathrm{f}}\left(z_{2}^{\mathrm{f}}\right) \in \mathbb{R}^{4}$ is done for the desired $\dot{z}_{2}\left(t_{k}^{-}\right)$, this choice is defined given some desired foot impact velocity $-v_{p_{2}}$

$\dot{z}_{2}^{\mathrm{f}}\left(z_{2}^{\mathrm{f}}, v_{p_{2}}\right):=\operatorname{Arg} \min _{\dot{z}_{2}}\left\|\dot{z}_{2}\right\|^{2} \quad$ under $\frac{\partial y_{p_{2}}}{\partial z_{2}}\left(z_{2}^{\mathrm{f}}\right) \dot{z}_{2}=-v_{p_{2}}$

$$
=-\left[\frac{\partial y_{p_{2}}}{\partial z_{2}}\left(z_{2}^{\mathrm{f}}\right)\right]^{\mathrm{T}} v_{p_{2}} /\left\|\frac{\partial y_{p_{2}}}{\partial z_{2}}\left(z_{2}^{\mathrm{f}}\right)\right\|^{2}
$$

where $y_{p_{2}}\left(z_{2}^{\mathrm{f}}\right)$ is the $y$-coordinate of the swing foot. Therefore, $\dot{z}_{2}^{\mathrm{f}}$ is clearly the minimum norm velocity vector that corresponds to some impact velocity $-v_{p_{2}}$. Once this choice is done, a final desired "just before impact" sub-state $\left(z_{2}, \dot{z}_{2}\right) \in \mathbb{R}^{8}$ is completely defined by the choice of $z_{2}^{\mathrm{f}} \in \mathbb{R}^{4}$.

In what follows, the following notations are used

$$
\begin{aligned}
& \mathscr{Z}_{2}:=\left(\begin{array}{c}
z_{2} \\
\dot{z}_{2}
\end{array}\right) \in \mathbb{R}^{8} ; \quad \mathscr{Z}_{2}^{\mathrm{f}}:=\left(\begin{array}{c}
z_{2}^{\mathrm{f}} \\
\dot{z}_{2}^{\mathrm{f}}\left(z_{2}^{\mathrm{f}}, v_{p_{2}}\right)
\end{array}\right) \in \mathbb{R}^{8} ; \\
& \mathscr{Z}_{1}:=\left(\begin{array}{c}
q_{1} \\
\dot{q}_{1}
\end{array}\right) \in \mathbb{R}^{2}
\end{aligned}
$$

Now, during the step, let us denote by $\eta>0$ the remaining 344 time before impact. One has the following dynamic for $\eta$

$\dot{\eta}=-1+\delta(\eta) \cdot t_{f}$

where $\delta(\cdot)$ is the generalized impulse function. Consider a control sampling period $\tau_{c}>0$ such that $t_{f} / \tau_{c}=N_{c} \in \mathbb{N}$ $\left(N_{c}\right.$ : is also a design parameter).

Basically, a problem of synchronizing the sampling times to the impact times could appear when impact is either detected prematurely (i.e. before the expected instant) or detected with a delay (i.e. after the expected instant). Since RABBIT PROTOTYPE feet are equipped with switches, the impact instant could easily be detected. This situation is managed as indicated in Section 4.3 concerning implementation issues.

Let us use the following notation to refer to decision instants [4] on the interval $\left[t_{k}, t_{k+1}\right]$

$\tau_{k}^{i}=t_{k}+i \tau_{c} ; \quad i \in\left\{0, \ldots, N_{c}-1\right\} ; \quad k \in \mathbb{N}$

During the step, at each decision instant $\tau_{k}^{i}$, a $p$-parameterized reference trajectory ${ }^{4}$

$\mathscr{Z}_{2}^{\text {ref }}\left(\tau^{\prime}, \mathscr{Z}_{2}\left(\tau_{k}^{i}\right), \mathscr{Z}_{2}^{\mathrm{f}}, \eta\left(\tau_{k}^{i}\right), p\right) ; \quad \tau^{\prime} \in\left[\tau_{k}^{i}, t_{k+1}\right] ; \quad p \in \mathscr{P}$

is defined that satisfies for all parameter value $p \in \mathscr{P}$ the following boundary (initial and final) conditions

$\mathscr{Z}_{2}^{\text {ref }}\left(\tau_{k}^{i}, \mathscr{Z}_{2}\left(\tau_{k}^{i}\right), \mathscr{Z}_{2}^{\mathrm{f}}, \eta\left(\tau_{k}^{i}\right), p\right)=\mathscr{Z}_{2}\left(\tau_{k}^{i}\right)$

$\mathscr{Z}_{2}^{\text {ref }}\left(t_{k+1}, \mathscr{Z}_{2}\left(\tau_{k}^{i}\right), \mathscr{Z}_{2}^{\mathrm{f}}, \eta\left(\tau_{k}^{i}\right), p\right)=\mathscr{Z}_{2}^{\mathrm{f}}$

namely, the reference trajectory $\mathscr{Z}_{2}^{\text {ref }}\left(\cdot, \mathscr{Z}_{2}\left(\tau_{k}^{i}\right), \mathscr{Z}_{2}^{\mathrm{f}}, \eta\left(\tau_{k}^{i}\right), p\right)$ is updated at each decision instant $\tau_{k}^{i}$ to start at the present value $\mathscr{Z}_{2}\left(\tau_{k}^{i}\right)$, and to join the desired final value $\mathscr{Z}_{2}^{\mathrm{f}}$ just before next impact.

It is worth noting that $p \in \mathscr{P}$ is the remaining free parameter, once the constraints (19) and (20) have been structurally imposed, on some initial parameterization. This is typically easy to realize with polynomial parameterization [9] of trajectories since (19) and (20) are linear constraints in the polynomial coefficients.

A relevant question is: how to choose $p \in \mathscr{P}$ ?

The role of $p$ is clearly to optimize the behavior of the indirectly controlled sub-state $\mathscr{Z}_{1}$. Indeed, imagine that a perfect tracking of the reference trajectory $\mathscr{Z}_{2}^{\text {ref }}\left(\cdot, \mathscr{Z}_{2}\left(\tau_{k}^{i}\right)\right.$, $\left.\mathscr{Z}_{2}^{\mathrm{f}}, \eta\left(\tau_{k}^{i}\right), p\right)$ is performed over $\left[\tau_{k}^{i}, t_{k+1}\right]$. What are the consequences of such tracking on the value of both $\mathscr{Z}_{1}$ and $\mathscr{Z}_{2}$ just before the $(k+1)$ impact?

- For $\mathscr{Z}_{2}$, one would clearly have, because of the perfect tracking [see (20)]

$$
\mathscr{Z}_{2}\left(t_{k+1}^{-}\right)=\mathscr{Z}_{2}^{\mathrm{f}}
$$

- For the $\mathscr{Z}_{1}$ dynamic, let us consider the torso equation extracted from the dynamic model (1), and given by

\footnotetext{
${ }^{4}$ In [16] for instance such trajectories are generated using Van der Pol oscillators.
} 


$$
\begin{aligned}
\left(\frac{1}{4} m_{1} l_{1}^{2}+I_{1}\right) \ddot{q}_{1}= & \frac{1}{2} m_{1} l_{1} \cos \left(q_{1}\right) \ddot{x} \\
& +\frac{1}{2} m_{1} l_{1} \sin \left(q_{1}\right)(\ddot{y}+g)-u_{1}-u_{2}
\end{aligned}
$$

where $m_{1}$ is the mass of the torso, $l_{1}$ its length, $u_{1}$ and $u_{2}$ are the torques of the femurs.

Using Eqs. (4) and (5) and notations (16), this dynamic should be written

$\dot{\mathscr{Z}}_{1}=f\left(\mathscr{Z}_{1}, \mathscr{Z}_{2}, u\right)$

The closed-loop system is obtained by state feedback, that is $u=K\left(\mathscr{Z}, \mathscr{Z}_{2}^{\text {ref }}\right)$, therefore equation (23) could be rewritten as

$$
\dot{\mathscr{Z}}_{1}=f\left(\mathscr{Z}_{1}, \mathscr{Z}_{2}, \mathscr{Z}_{2}^{\text {ref }}\right)
$$

Under the assumption of perfect tracking, by replacing $\mathscr{Z}_{2}$ in (24) by the reference trajectory $\mathscr{Z}_{2}^{\text {ref } 5}$ one obtains:

$$
\dot{\mathscr{Z}}_{1}=f\left(\mathscr{Z}_{1}, \mathscr{Z}_{2}^{\text {ref }}\right)=f\left(\mathscr{Z}_{1}, \mathscr{Z}_{2}\left(\tau_{k}^{i}\right), \mathscr{Z}_{2}^{\mathrm{f}}, p\right)
$$

and integrating (25) starting from the initial condition $\left(\tau_{k}^{i}, \mathscr{Z}_{1}\left(\tau_{k}^{i}\right)\right)$ gives the predicted value of $\mathscr{Z}_{1}\left(t_{k+1}^{-}\right)$just before next impact. This can be rewritten formally as follows $\left(\eta\left(\tau_{k}^{i}\right)=t_{k+1}-\tau_{k}^{i}\right)$

$$
\widehat{\mathscr{Z}}_{1}\left(t_{k+1}^{-} \mid \tau_{k}^{i}\right)=\Psi\left(\mathscr{Z}_{1}\left(\tau_{k}^{i}\right), \mathscr{Z}_{2}\left(\tau_{k}^{i}\right), \mathscr{Z}_{2}^{\mathrm{f}}, \eta\left(\tau_{k}^{i}\right), p\right)
$$

and using the impact equation (cf. Eq. (14)) together with the predicted values (21) and (26) one can derive an expression of the predicted value of $\mathscr{Z}_{1}$ just after impact

$$
\widehat{\mathscr{Z}}_{1}\left(t_{k+1}^{+} \mid \tau_{k}^{i}\right)=\Psi^{+}\left(\mathscr{Z}_{1}\left(\tau_{k}^{i}\right), \mathscr{Z}_{2}\left(\tau_{k}^{i}\right), \mathscr{Z}_{2}^{\mathrm{f}}, \eta\left(\tau_{k}^{i}\right), p\right)
$$

The value of the reference trajectory's parameter $p\left(\tau_{k}^{i}\right)$ is then given by the optimal solution of the following quadratic optimization problem

$\hat{p}\left(\tau_{k}^{i}\right)=\min _{p \in \mathscr{P}}\left\|\hat{\mathscr{Z}}_{1}\left(t_{k+1}^{+} \mid \tau_{k}^{i}\right)-\mathscr{Z}_{1}^{\mathrm{f}}\right\|_{Q}^{2}$ subject to

$$
C\left(\mathscr{Z}_{2}\left(\tau_{k}^{i}\right), \mathscr{Z}_{2}^{\mathrm{f}}, p\right)>0 ; \quad Q \in \mathbb{R}^{2 \times 2} \quad Q>0
$$

where

- $\mathscr{Z}_{1}^{\mathrm{f}} \in \mathbb{R}^{2}$ is some desired value just after the impact. This value (together with $\mathscr{Z}_{2}^{\mathrm{f}}$ ) defines the limit cycle one aims to establish.

- $C\left(\mathscr{Z}_{2}\left(\tau_{k}^{i}\right), \mathscr{Z}_{2}^{\mathrm{f}}, p\right)>0$ is a constraint expressing non penetration condition. This can be for instance

$$
C\left(\mathscr{Z}_{2}\left(\tau_{k}^{i}\right), \mathscr{Z}_{2}^{\mathrm{f}}, p\right):=\min _{\tau^{\prime} \in\left[\tau_{k}^{i}, t_{k+1}-\epsilon\right]} y_{p_{2}}\left(\tau^{\prime}, \mathscr{Z}_{2}\left(\tau_{k}^{i}\right), \mathscr{Z}_{2}^{\mathrm{f}}, p\right)
$$

444 for some small $\epsilon>0$.
To summarize, ${ }^{6}$ during the step, at each decision instant $\tau_{k}^{i}$ with $i<N_{c}-1$ the reference trajectory

\footnotetext{
${ }^{5}$ Recall that $\mathscr{Z}_{2}^{\text {ref }}$ depends on $\mathscr{Z}_{2}\left(\tau_{k}^{i}\right), \mathscr{Z}_{2}^{\mathrm{f}}$, and $p$.

${ }^{6}$ A chart flow better illustrating the principle of the approach is given in Section 4.3.
}

$\mathscr{Z}_{2}^{\text {ref }}\left(\tau^{\prime}, \mathscr{Z}_{2}\left(\tau_{k}^{i}\right), \mathscr{Z}_{2}^{\mathrm{f}}, \eta\left(\tau_{k}^{i}\right), \hat{p}\left(\tau_{k}^{i}\right)\right) ; \quad \tau^{\prime} \in\left[\tau_{k}^{i}, t_{k+1}\right]$

is defined on the completely controlled variables (actuated joints) and tracked using a nonlinear time varying feedback during the time interval $\left[\tau_{k}^{i}, \tau_{k}^{i+1}\right]$. At the next decision instant $\tau_{k}^{i+1}$ a new reference trajectory

$\mathscr{Z}_{2}^{\text {ref }}\left(\tau^{\prime}, \mathscr{Z}_{2}\left(\tau_{k}^{i+1}\right), \mathscr{Z}_{2}^{\mathrm{f}}, \eta\left(\tau_{k}^{i+1}\right), \hat{p}\left(\tau_{k}^{i+1}\right)\right) ; \quad \tau^{\prime} \in\left[\tau_{k}^{i}, t_{k+1}\right]$

is defined, based on the new measurements and is tracked during the time interval $\left[\tau_{k}^{i+1}, \tau_{k}^{i+2}\right]$ and the scheme is repeated until the impact instant. This defines a predictive control scheme in which the open-loop auxiliary optimization problem is given by (28). The solution of such optimization problem is performed using the DBCPOL function from the IMSL math library of Digital Fortran 5.0.

\section{Stability and implementation issues}

The stability can be investigated using the Poincarés section [17] just before the impact, namely at instants $t_{k}^{-}$. Indeed, if this discrete-time map converges, then a cyclic trajectory results (see Figs. 5 and 6). To study the stability of the Poincaré's map, note that, by definition of the predictive control strategy depicted in the previous section, one clearly has

$\mathscr{Z}_{2}\left(t_{k}^{-}\right)=\mathscr{Z}_{2}^{\mathrm{f}}$

where $\mathscr{Z}_{2}^{\mathrm{f}}$ is the desired final "just before impact" configuration defined by (15) and (16) and depending only on the desired final position $z_{2}^{\mathrm{f}}$. Consequently, the overall stability depends on the stability of the sequence

$\left(\mathscr{Z}_{1}\left(t_{k}^{-}\right)\right)_{k \in \mathbb{N}}$

under the constraint (30).

\subsection{Stability definition}

As it can be easily understood from Figs. 5 and 6, an asymptotically stable $k_{0}$-cyclic trajectory on the whole state results whenever the following property holds for the closed-loop system's behavior:

$\left\|\mathscr{Z}_{1}\left(t_{(j+1) k_{0}}^{-}\right)-\mathscr{Z}_{1}^{\mathrm{f}}\right\|_{Q}^{2} \leqslant \mu\left\|\mathscr{Z}_{1}\left(t_{j k_{0}}^{-}\right)-\mathscr{Z}_{1}^{\mathrm{f}}\right\|_{Q}^{2} ; \quad \mu<1$

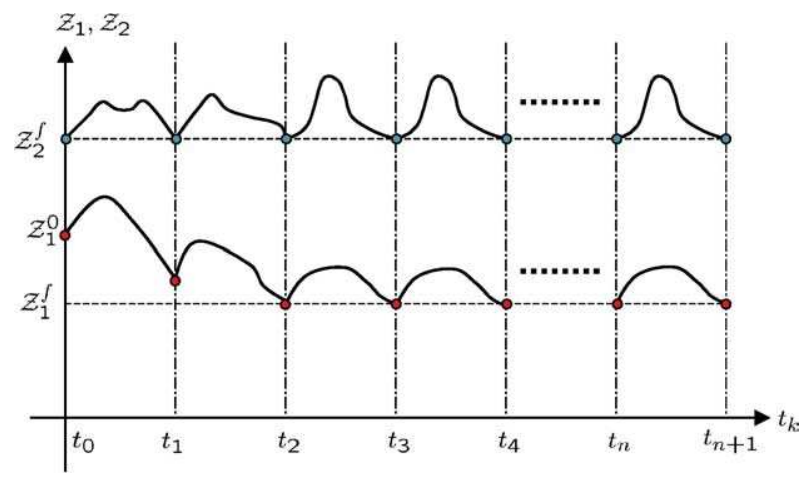

Fig. 5. Stability illustration $\left(k_{0}=1\right)$. 


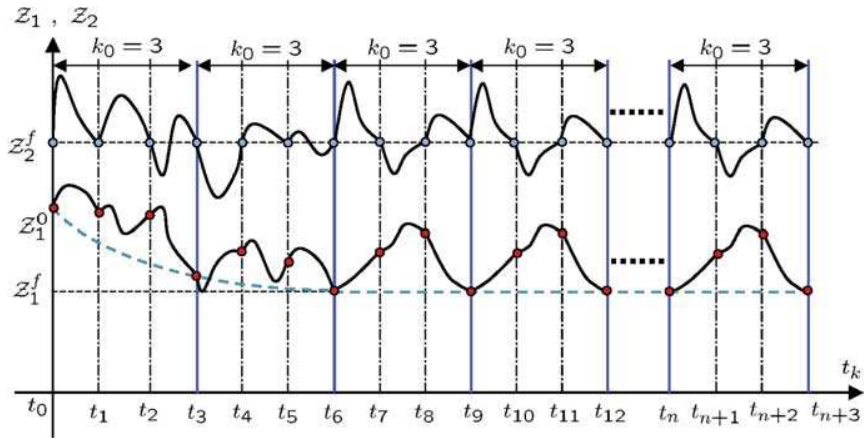

Fig. 6. Stability illustration $\left(k_{0}=3\right)$.

similarly, a neighborhood of a $k_{0}$ cyclic trajectory on the whole is asymptotically stabilized whenever the following property holds for some small $\varepsilon>0$,

$\lim _{j \rightarrow \infty}\left\|\mathscr{Z}_{1}\left(t_{j k_{0}}^{-}\right)-\mathscr{Z}_{1}^{\mathrm{f}}\right\|_{Q}^{2} \leqslant \varepsilon$

The aim of the following section is to give sufficient conditions under which one of the above conditions is satisfied for some $k_{0} \in \mathbb{N}$ with a graphical tools enabling a concrete evaluation of the associated conditions (see Proposition 1 hereafter).

\subsection{Stability result}

Now let $\mathscr{Z}_{1}\left(t_{k}^{-}\right)$be given. Using (30) and the impact map (14), the value of the whole $\mathscr{Z}\left(t_{k}^{+}\right)$just after the impact can be computed and the predictive control closed-loop trajectories may be predicted over $\left[t_{k}^{+}, t_{k+1}^{-}\right]$. Therefore, the predicted value of $\mathscr{Z}_{1}\left(t_{k+1}^{-}\right)$just before the next impact is only function of $\mathscr{Z}_{1}\left(t_{k}^{-}\right), N_{c}$ and $\mathscr{Z}^{\mathrm{f}}:=\left(\mathscr{Z}_{1}^{\mathrm{f}}, \mathscr{Z}_{2}^{\mathrm{f}}\right)$, namely

$$
\mathscr{Z}_{1}\left(t_{k+1}^{-}\right)=: \Gamma\left(\mathscr{Z}_{1}\left(t_{k}^{-}\right), \mathscr{Z}^{\mathrm{f}}, N_{c}\right)
$$

which is a discrete-time autonomous system (for fixed $\mathscr{Z}^{\mathrm{f}}$ and $N_{c}$ ) in the sub-state $\mathscr{Z}_{1}$ for which stability is to be investigated. More generally, the following multi-step map is particularly relevant to assess the stability of the above predictive control scheme, namely,

$\mathscr{Z}_{1}\left(t_{k+k_{0}}^{-}\right)=: \Gamma^{k_{0}}\left(\mathscr{Z}_{1}\left(t_{k}^{-}\right), \mathscr{Z}^{\mathrm{f}}, N_{c}\right)$

where $\Gamma^{k_{0}}$ is obtained by repetitive application of $\Gamma(\cdot)$. Note that this map is easily computable by simulating $k_{0}$ steps under the closed-loop feedback law explained in the previous section. It is worth noting that such computations are to be done off-line for stability investigations. The on-line feedback however is still based on one-step scalar optimization as explained in the preceding section. The whole closed-loop system stability analysis is based on the following proposition

\section{Proposition 1}

1. If for some $\left(k_{0}, N_{c}\right) \in \mathbb{N} \times \mathbb{N}$, there is $\varrho>0$ such that

$$
\sup _{\left\|\mathscr{L}_{1}-\mathscr{Z}_{1}^{\mathrm{f}}\right\|_{Q}^{2} \leqslant \varrho}\left\|\Gamma^{k_{0}}\left(\mathscr{Z}_{1}, \mathscr{Z}^{\mathrm{f}}, N_{c}\right)-\mathscr{Z}_{1}^{\mathrm{f}}\right\|_{Q}^{2} \leqslant \varrho
$$

then the predictive control closed-loop leads to a stable walk for all initial conditions belonging to the set

$\mathscr{C}_{0}:=\left\{\mathscr{Z}=\left(\begin{array}{l}\mathscr{Z}_{1} \\ \mathscr{Z}_{2}^{\text {f }}\end{array}\right)\right.$ s.t. $\left.\mathscr{Z}_{1} \in \mathbb{M}_{\varrho}\right\}$

where for all $\varrho \geqslant 0, \mathbb{M}_{\varrho}:=\left\{\mathscr{Z}_{1} \mid\left\|\mathscr{Z}_{1}-\mathscr{Z}_{1}^{\mathrm{f}}\right\|_{Q}^{2} \leqslant \varrho\right\}$.

2. If in addition, the following condition holds for some $\mu \in[0,1[$

For all $0<r<\varrho \quad \psi\left(r, N_{c}, k_{0}\right)$

$$
:=\sup _{\left\|\mathscr{L}_{1}-\mathscr{Z}_{1}^{\mathrm{f}}\right\|_{Q}^{2}=r}\left\|\Gamma^{k_{0}}\left(\mathscr{Z}_{1}, \mathscr{Z}^{\mathrm{f}}, N_{c}\right)-\mathscr{Z}_{1}^{\mathrm{f}}\right\|_{Q}^{2} \leqslant \mu \cdot r
$$

then the closed-loop trajectories asymptotically converges to a stable limit cycle of length $k_{0}$ defined by the pair $\left(\mathscr{Z}_{1}^{\mathrm{f}}, \mathscr{Z}_{2}^{\mathrm{f}}\right)$ for all initial conditions in $\mathscr{C}_{0}$.

3. If (35) holds, and if (37) holds for all $r \in[\varepsilon, \varrho]$ and furthermore,

$$
\sup _{\left\|\mathscr{V}_{1}-\mathscr{Z}_{1}^{\mathrm{f}}\right\|_{Q}^{2} \leqslant \varepsilon}\left\|\Gamma^{k_{0}}\left(\mathscr{Z}_{1}, \mathscr{Z}^{\mathrm{f}}, N_{c}\right)-\mathscr{Z}_{1}^{\mathrm{f}}\right\|_{Q}^{2} \leqslant \varepsilon ; \quad \varepsilon<\varrho
$$

(see Fig. 7 for a typical situation) then the set

$$
\mathscr{C}_{1}:=\left\{\mathscr{Z}=\left(\begin{array}{l}
\mathscr{Z}_{1} \\
\mathscr{Z}_{2}^{\text {f }}
\end{array}\right) \text { s.t. } \mathscr{Z}_{1} \in \mathbb{M}_{\varepsilon}\right\}
$$

is invariant and attractive for all initial conditions in $\mathscr{C}_{0}$ (an \&-neighborhood of the limit cycle is reached).

\section{Proof}

1. Straightforward since condition (35) implies that the set 556 $\mathbb{M}_{\varrho}$ is invariant under the composed map $\Gamma^{k_{0}}\left(\cdot, \mathscr{Z}^{\mathrm{f}}, N_{c}\right)$, more precisely

$\left\{\mathscr{Z}_{1} \in \mathbb{M}_{\varrho}\right\} \Rightarrow\left\{\Gamma^{k_{0}}\left(\mathscr{Z}_{1}, \mathscr{Z}^{\mathrm{f}}, N_{c}\right) \in \mathbb{M}_{\varrho}\right\}$

Therefore, starting from some initial value $\mathscr{Z}_{1}^{0} \in \mathbb{M}_{\varrho}, \quad 561$ the sequence

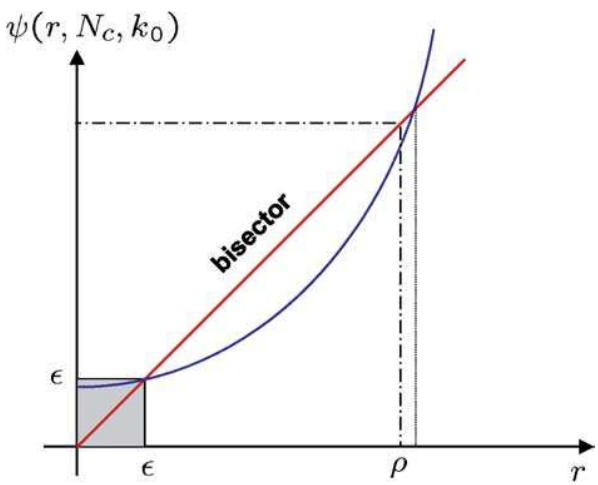

Fig. 7. Typical situation where (35), (37) and (38) hold (point 3 of Proposition 1). 
$\left(\mathscr{Z}_{1}\left(t_{j k_{0}}^{-}\right)\right)_{j=1}^{\infty}$

belongs to the compact set $\mathbb{M}_{\varrho}$.

566 2. Condition (37) implies that for all $j \in \mathbb{N}$, one has

$\left\|\mathscr{Z}_{1}\left(t_{(j+1) k_{0}}^{-}\right)-\mathscr{Z}_{1}^{\mathrm{f}}\right\|_{Q}^{2} \leqslant \mu\left\|\mathscr{Z}_{1}\left(t_{j k_{0}}^{-}\right)-\mathscr{Z}_{1}^{\mathrm{f}}\right\|_{Q}^{2}$

570

(where $\mu<1$ ). Accordingly, by recurrence, one obtains (for $m \in \mathbb{N}$ )

$$
\left\|\mathscr{Z}_{1}\left(t_{(j+m) k_{0}}^{-}\right)-\mathscr{Z}_{1}^{\mathrm{f}}\right\|_{Q}^{2} \leqslant \mu^{m}\left\|\mathscr{Z}_{1}\left(t_{j k_{0}}^{-}\right)-\mathscr{Z}_{1}^{\mathrm{f}}\right\|_{Q}^{2}
$$

which implies that

$\lim _{j \rightarrow \infty} \mathscr{Z}_{1}\left(t_{j k_{0}}^{-}\right)=\mathscr{Z}_{1}^{\mathrm{f}}$

This shows that the closed-loop trajectories tends to a limit cycle of length $k_{0}$ defined by the pair of desired values $\left(\mathscr{Z}_{1}^{\mathrm{f}}, \mathscr{Z}_{2}^{\mathrm{f}}\right)$.

3. Using the same argumentation as in the last point, Eq. (41) may be rewritten for all $\mathscr{Z}_{1}\left(t_{j k_{0}}^{-}\right)$that lies in $\mathbb{M}_{\varrho} \backslash \mathbb{M}_{\varepsilon}$. This proves that $\mathbb{M}_{\varepsilon}$ is attractive. Furthermore, $\mathbb{M}_{\varepsilon}$ is invariant.

Note again that the investigation of (35)-(38) may be done off-line simultaneously and in a deterministic way by solving the following two-dimensional optimization problem

$\psi\left(r, N_{c}, k_{0}\right):=\sup _{\left\|\mathscr{Z}_{1}-\mathscr{Z}_{1}^{\mathrm{f}}\right\|_{Q}^{2}=r}\left\|\Gamma^{k_{0}}\left(\mathscr{Z}_{1}, \mathscr{Z}^{\mathrm{f}}, N_{c}\right)-\mathscr{Z}_{1}^{\mathrm{f}}\right\|_{Q}^{2}$

for increasing values of $r$ and check wether the curve so obtained [see Fig. 7] satisfies (35)-(38) for some $\varrho>0$ and $\varepsilon>0$. The whole procedure may be repeated for different values of $k_{0}$. Concrete examples of such plots are given in Section 5 for a specific choice of the design parameters $\mathscr{Z}^{\mathrm{f}}, Q$ and $N_{c}$. It is then shown that the condition of point 3. of Proposition 1 are satisfied for $k_{0}=3$ (see Fig. 11) while it is not satisfied for $k_{0}=1,2$. This shows the need for non trivial multi-step map (34) in establishing the stability of the underlying closed-loop behaviour.

\subsection{Implementation issues}

The reference trajectories (18) are implemented using Matlab cubic spline interpolation functions with various end-conditions. They are parameterized with a free parameter $p$ which should be computed by solution of the optimization problem (28). The use of the cubic spline functions requires the definition of the end-conditions, in our case they are given by

- initial-time conditions, given by Eq. (19),

- intermediate-time conditions, given by the parameter $p$ to be computed,

- final-time conditions, given by Eq. (20).

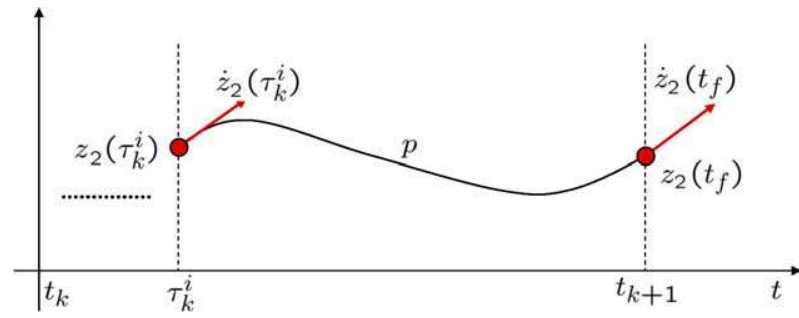

Fig. 8. The directly controlled variables trajectories.

The obtained trajectory, that satisfy these constraints, 614 may be illustrated in Fig. 8 .

The used subroutines provide the cubic spline interpolant, which should be used to evaluate the trajectory and its derivatives, at each instant $\tau^{\prime}$. The switching to a new 6 step is closely related to the impact occurrence. The implemented simulator (using vi sual Fortran 5.0 and Matlab 6.5 softwares) switches to a new step once it detects an impact, therefore three possible cases could be underlined

1. The biped walks without external disturbances, the 624 dynamic model is perfect, as well as the tracking of 625 the optimal reference trajectories.

2. During walking, because of external disturbances, model imperfections, or obstacles, the impact is detected prematurely.

3. The biped, during walking is subject to external disturbances, model imperfections, or environment changes, as a consequence the impact is not detected at the expected time (instant).

How the control system would react?

In the first case there is no problem, the whole closed- 636 loop system behavior looks like the predicted one. In the 637 second case, when the impact is detected, the reached con- 638 figuration just after the impact is considered as an initial 639 configuration, the final desired configuration is then com- 640 puted, and a new step starts (illustrated in simulation 4). 641 While in the third case, the impact is not detected at the 642 expected instant, so the control system proceed to an 643 extrapolation of the computed trajectories (using the 644 Matlab PPVAL function) until the occurrence of the forth- 645 coming impact. The whole control approach is summarized 646 in the diagram depicted in Fig. 9 that illustrates how it 647 works.

\section{Illustrative simulations}

Consider the biped robot model (7) and (14) with the parameters summarized in Table 1. The control parameter 6 $N_{\mathrm{c}}=1$ is used in the following simulations, enabling a large admissible on-line computation time. Indeed, with this 6 choice, $\tau_{\mathrm{c}}=t_{\mathrm{f}}$ and the trajectories being tracked during the step are updated just after each impact. The following 


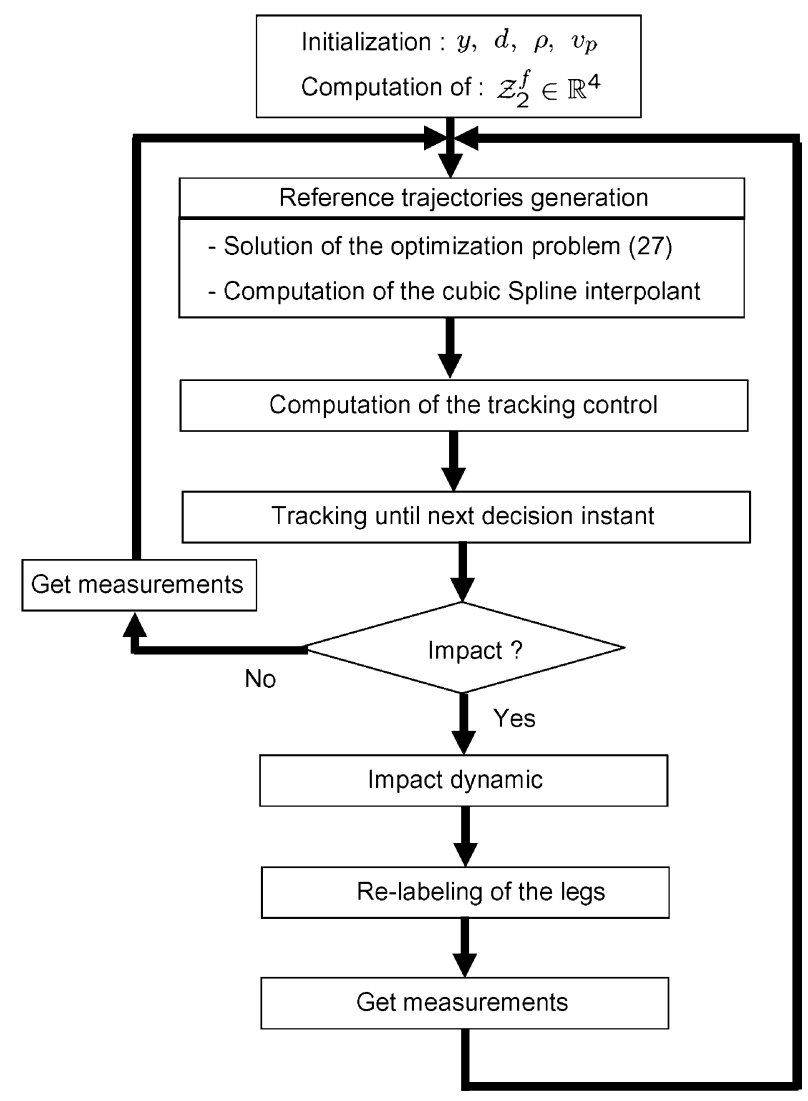

Fig. 9. Algorithm of the approach.

Table 1

The model parameters

\begin{tabular}{llll}
\hline Parameter & Mass $(\mathrm{kg})$ & Length $(\mathrm{m})$ & Inertia $\left(\mathrm{kg} \mathrm{m}^{2}\right)$ \\
\hline Torso & 20 & 0.625 & 2.22 \\
Femur & 6.8 & 0.4 & 1.08 \\
Tibia & 3.2 & 0.4 & 0.93 \\
\hline
\end{tabular}

choice of the parameter $p$ is used in the definition of the predictive control law (see Section 3):

$p\left(t_{k}\right):=\pi-q_{31}\left(t_{k}+t_{f} / 2\right)$

Remark 2. The proposed choice of the optimization parameter represents the angular position of the femur of the swing leg at median instant between two impacts. This is a particular choice among many others, for instance one can imagine any free parameter on the trajectories of the actuated coordinates or their derivatives, it can also be one of the configuration parameters ( $\rho$ for instance).

Two simulation scenarios are proposed:

- The first one shows how the biped reaches a stable walk with constant mean velocity starting from rest.
- The second one illustrates the transition between several walks with different mean walking velocities.

For robustness evaluation of the proposed controller, 675 two scenarios are investigated, namely

- Robustness against uncertainties in the robot model parameters.

- Robustness against ground irregularities.

Let us first illustrate how $z_{2}^{\mathrm{f}}$ is chosen by means of a reduced dimensional parameterization. The way such choice of $z_{2}^{\mathrm{f}}$ may be made optimal in some sense is beyond the scope of the present paper and will be investigated in later works.

682

\subsection{Reduced dimensional parameterization of the position vector $z_{2}$}

Consider the instantaneous double support configuration. The position vector $z_{2}:=\left(\begin{array}{llll}q_{31} & q_{41} & q_{32} & q_{42}\end{array}\right)^{\mathrm{T}}$ is defined by three simple parameters, namely $y, d$ and $\rho$ that are illustrated in Fig. 10.

Indeed, simple computations give

$$
\left\{\begin{array}{l}
q_{31}=\pi-\arctan \left(\frac{\rho d}{y}\right)-\varphi_{31} \\
q_{32}=\pi+\arctan \left(\frac{(1-\rho) d}{y}\right)-\varphi_{32} \\
q_{41}=\pi-\varphi_{41}=\arccos \left(-\frac{l_{3}^{2}+l_{4}^{2}-\rho^{2} d^{2}-y^{2}}{2 l_{3} l_{4}}\right) \\
q_{42}=\pi-\varphi_{42}=\arccos \left(-\frac{l_{3}^{2}+l_{4}^{2}-(1-\rho)^{2} d^{2}-y^{2}}{2 l_{3} l_{4}}\right)
\end{array}\right.
$$

where

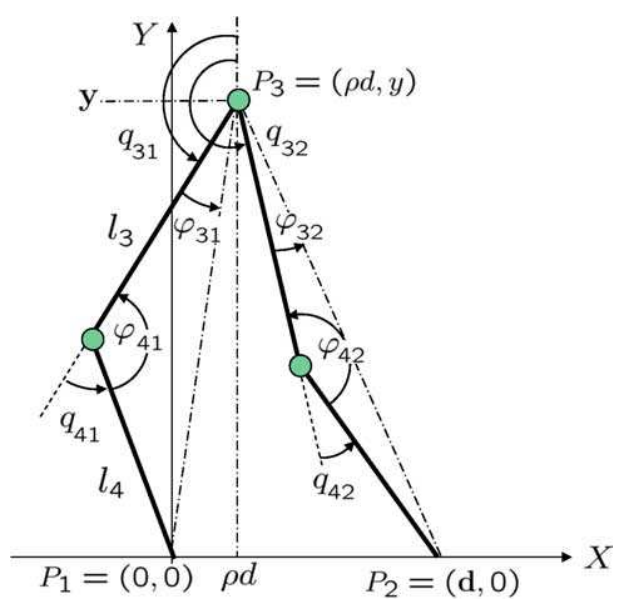

Fig. 10. Computation scheme for the position's reduced parameterization. 


$$
\left\{\begin{array}{l}
\varphi_{31}=\arccos \left(\frac{l_{3}^{2}-l_{4}^{2}+\rho^{2} d^{2}+y^{2}}{2 l_{3} \sqrt{\rho^{2} d^{2}+y^{2}}}\right) \\
\varphi_{32}=\arccos \left(\frac{l_{3}^{2}-l_{4}^{2}+(1-\rho)^{2} d^{2}+y^{2}}{2 l_{3} \sqrt{(1-\rho)^{2} d^{2}+y^{2}}}\right) \\
\varphi_{41}=\arccos \left(\frac{l_{3}^{2}+l_{4}^{2}-\rho^{2} d^{2}-y^{2}}{2 l_{3} l_{4}}\right) \\
\varphi_{42}=\arccos \left(\frac{l_{3}^{2}+l_{4}^{2}-(1-\rho)^{2} d^{2}-y^{2}}{2 l_{3} l_{4}}\right)
\end{array}\right.
$$

698 This enables a simple choice of the desired final configura699 tion just before the impact $z_{2}^{\mathrm{f}}$ using parameters that are di700 rectly linked to the mean velocity and the geometric 701 configuration [32].

\section{5.2. Simulation 1: cyclic forward walking starting}

703 from rest (standing position)

The aim of this simulation is to take the robot from a

$\psi\left(r, N_{c}, k_{0}\right)=\sup _{\left\|\mathscr{Z}_{1}-\mathscr{L}_{1}^{f}\right\|_{Q}^{2}=r}\left\|\Gamma^{k_{0}}\left(\mathscr{Z}_{1}, \mathscr{Z}^{\mathrm{f}}, N_{c}\right)-\mathscr{Z}_{1}^{\mathrm{f}}\right\|_{\mathcal{Q}}^{2}$

715 invoked in Proposition 1, for the two cases corresponding 716 to $k_{0}=1,2$. Note that: rest position to a constant speed periodic walking. The configuration $z_{2}^{\mathrm{f}}$ and the other control design parameters are summarized in Table 2.

\subsubsection{Stability analysis according to Proposition 1}

In this section, it is shown that under the feedback defined above, the sufficient conditions invoked in point 3. of Proposition 1 are satisfied. This can be verified on Fig. 11, that shows the multi-step map

- For $k_{0}=1$ the conditions of Proposition 1 are not satisfied. Higher values of $k_{0}$ must be investigated in order to prove stability of the closed-loop system. Recall that $k_{0}$ is only an analysis tool and not a design tool.

- For $k_{0}=2$, the conditions of point 3 of Proposition 1 are satisfied with $\varrho \approx 0.56$ and $\varepsilon \approx 0.08$, therefore for all initial conditions in the set $\mathscr{C}_{0}$ given by (36) with

Table 2

The approach's parameters description

\begin{tabular}{lll}
\hline & Significance & Value \\
\hline$t_{\mathrm{f}}$ & Step duration & $0.75 \mathrm{~s}$ \\
$y$ & Hips height & $y=0.775$ \\
$d$ & Step length & $0.3 \mathrm{~m}$ \\
$\rho$ & Hip's $x$ position w.r.t. step length & 0.5 \\
$v_{\mathrm{p}}$ & Foot impact velocity & $-0.25 \mathrm{~m} / \mathrm{s}$ \\
$\mathscr{Z}_{10}$ & Initial conditions on the torso & $(0,0)$ \\
$Q$ & Weighting matrix in optimization & $\left(\begin{array}{ll}1 & 0 \\
0 & 0\end{array}\right)$ \\
$v_{\text {mean }}$ & Mean walking velocity & $-0.4 \mathrm{~m} / \mathrm{s}$ \\
\hline
\end{tabular}

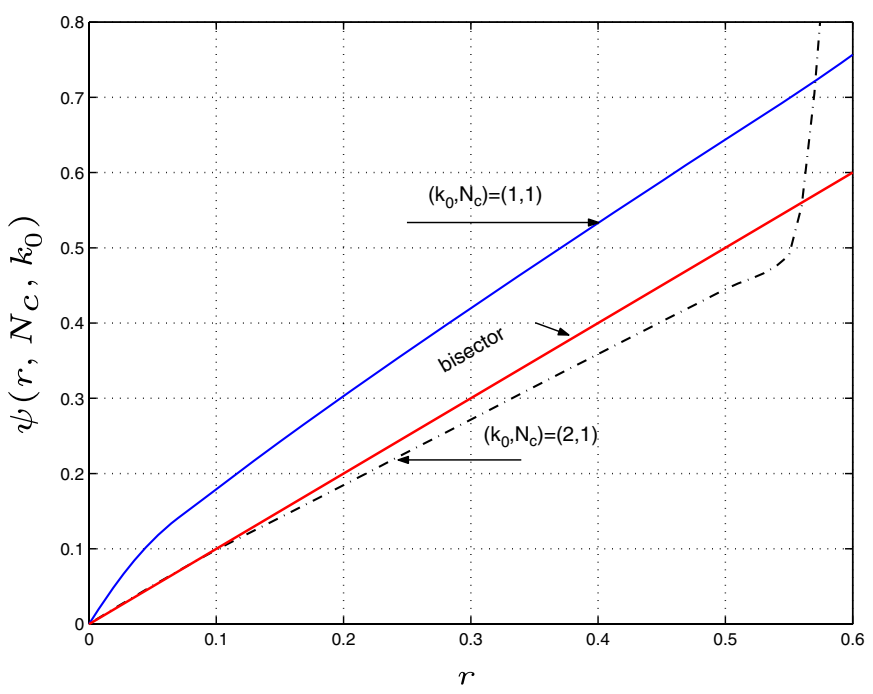

Fig. 11. (Sim 1) A stability analysis tool: the curve $\psi\left(r, N_{\mathrm{c}}, k_{0}\right)$ for different values of $k_{0}$.

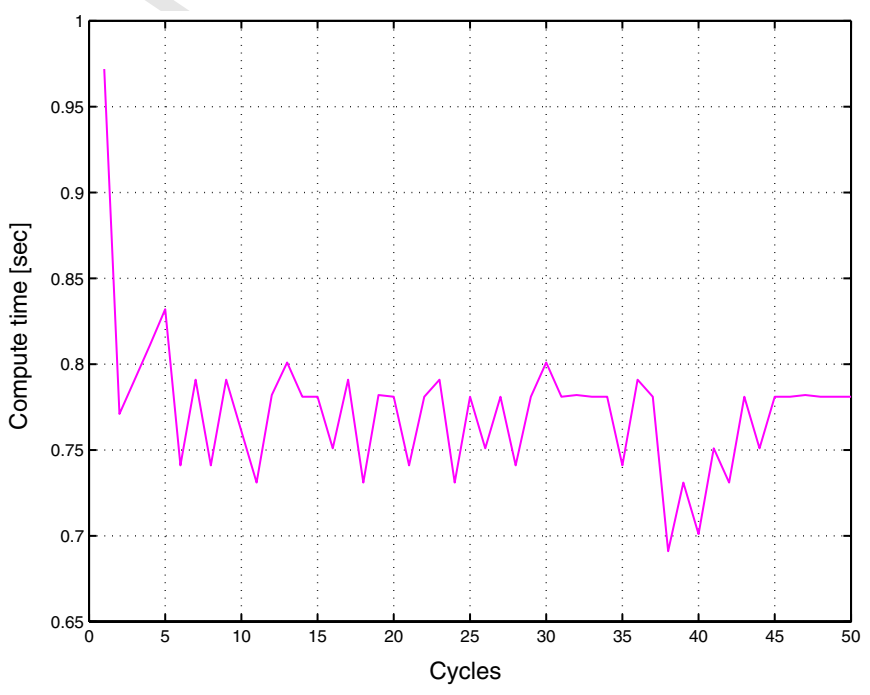

Fig. 12. (Sim 1) Evaluation of computation time.

$$
\mathbb{M}_{\varrho}:=\left\{\mathscr{Z}_{1} \mid\left\|\mathscr{Z}_{1}-\mathscr{Z}_{1}^{\mathrm{f}}\right\|_{Q}^{2} \leqslant 0.56\right\}
$$

the closed-loop trajectories impacts on the Poincaré sec- 726 tion converge to the invariant and attractive set $\mathscr{C}_{1}$ given by (39) with

$\mathbb{M}_{\varepsilon}:=\left\{\mathscr{Z}_{1} \mid\left\|\mathscr{Z}_{1}-\mathscr{Z}_{1}^{\mathrm{f}}\right\|_{Q}^{2} \leqslant 0.08\right\}$

which is a neighborhood of the desired limit cycle of 731 length 2 defined by $\mathscr{Z}_{1}^{\mathrm{f}}=0$.

This example shows clearly the need to the multi-step 733 stability analysis tool developed in Proposition 1, since 734 for $k_{0}<2$, stability cannot be claimed.

Remark 3. To give some concrete idea about how large is 736 the region of attraction, note that the set of initial 737 conditions $\mathbb{M}_{\varrho}$ leading to convergence to the neighborhood 738 
739 of the limit cycle corresponds, among others to the 740 following two initial conditions:

$742\left(q_{1}, \dot{q}_{1}\right)_{0}=\left( \pm 42.87^{\circ}, 0^{\circ} / \mathrm{s}\right)$

\section{5.2.2. More simulation results}

744
The behavior of the closed-loop system is to be illustrated through the following simulation results. In Fig. 13 the phase portrait $\left(q_{1}-\dot{q}_{1}\right)$ [16] of the unactuated coordinate (torso) is displayed, where we note the convergence to a neighborhood of a limit cycle of length 2 , which confirm the stability result discussed above. The mean walking velocity evolution is shown in Fig. 14 where the transition from a rest to the desired mean velocity stable walk can be observed. Note that the mean velocity is computed as the ratio between $\Delta x(k)$ and $t_{f}$ where $\Delta x(k)=x\left(t_{k}\right)-x\left(t_{k-1}\right)$. The position and velocity of the torso coordinate is shown in Fig. 15 where we note through its trajectory that it

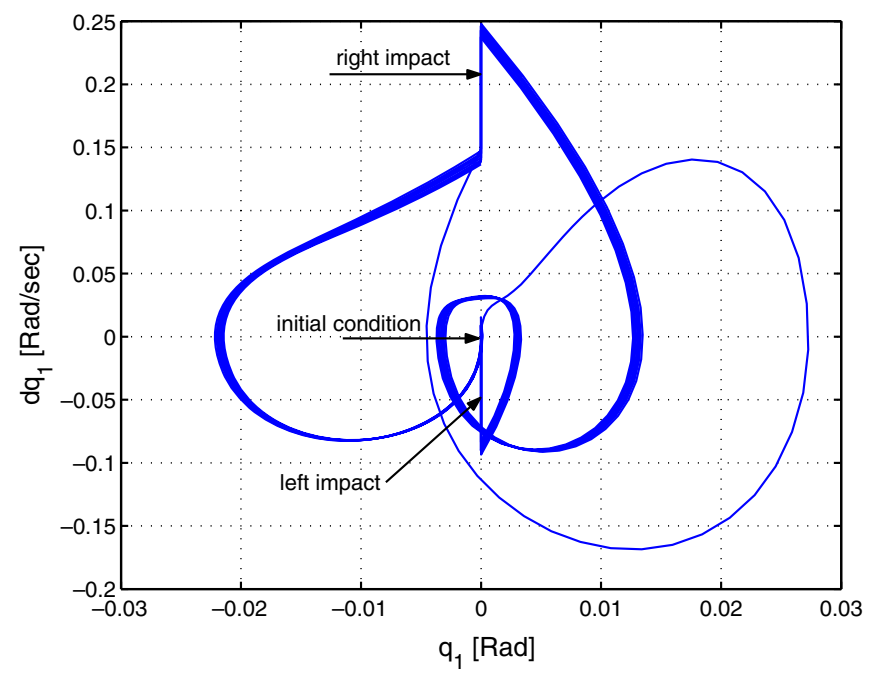

Fig. 13. (Sim 1) The phase portrait of the non actuated coordinate (torso).

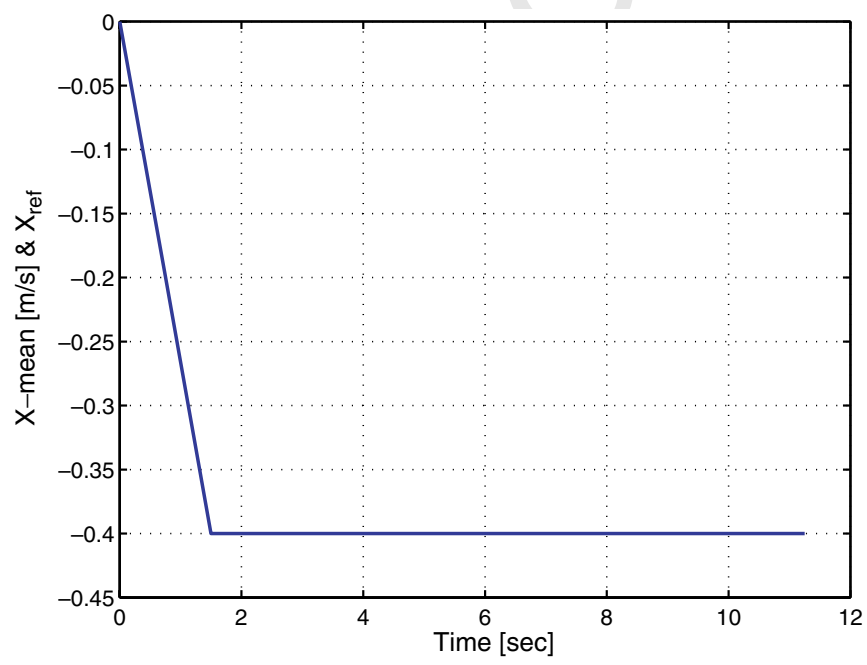

Fig. 14. (Sim 1) The mean walking velocity.
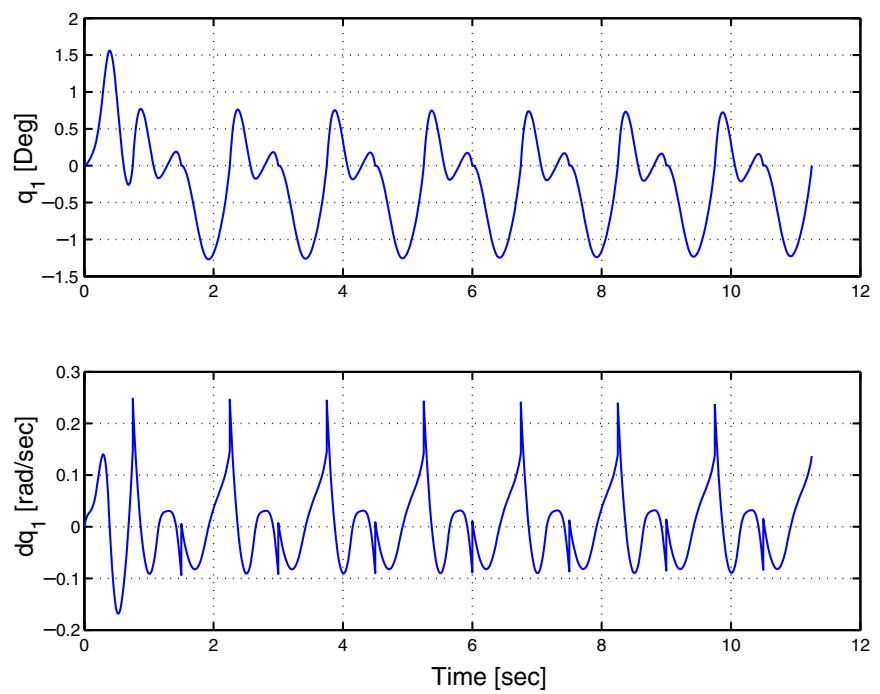

Fig. 15. (Sim 1) The torso position and velocity versus time.

remains close to the vertical. The cartesian coordinates (and their corresponding velocities) of the hips are depicted in Fig. 17 (for the $x$ coordinate) and in Fig. 18 (for the $y$ coordinate), furthermore the resulting trajectory of the hips in the plane $x-y$ is illustrated in Fig. 16 .

The system control inputs (i.e. joint torques) to be applied to the actuated joints are depicted in Fig. 19 for both femurs, and in Fig. 20 for both tibias. We note that RABBIT is equipped with dc motors of a maximum torque of $150 \mathrm{~N} \mathrm{~m}$, therefore according to the figures of the generated torques we conclude that this bound is largely satisfied, but it should also be checked that the power requirement remain within the admissible limit.

To check the admissibility of the actuators required power, the idea is to plot the angular velocity of the actuators versus their absolute torques, and check if the obtained curves remain within the admissible region given by the manufacturer of the actuators (DC motors). If it is

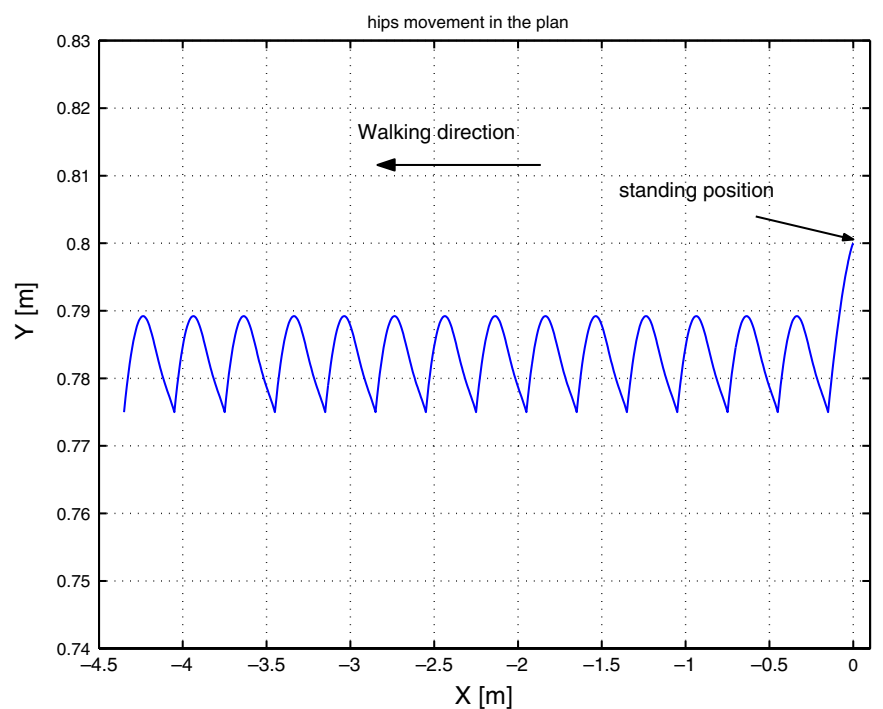

Fig. 16. (Sim 1) The hips movement trajectory. 

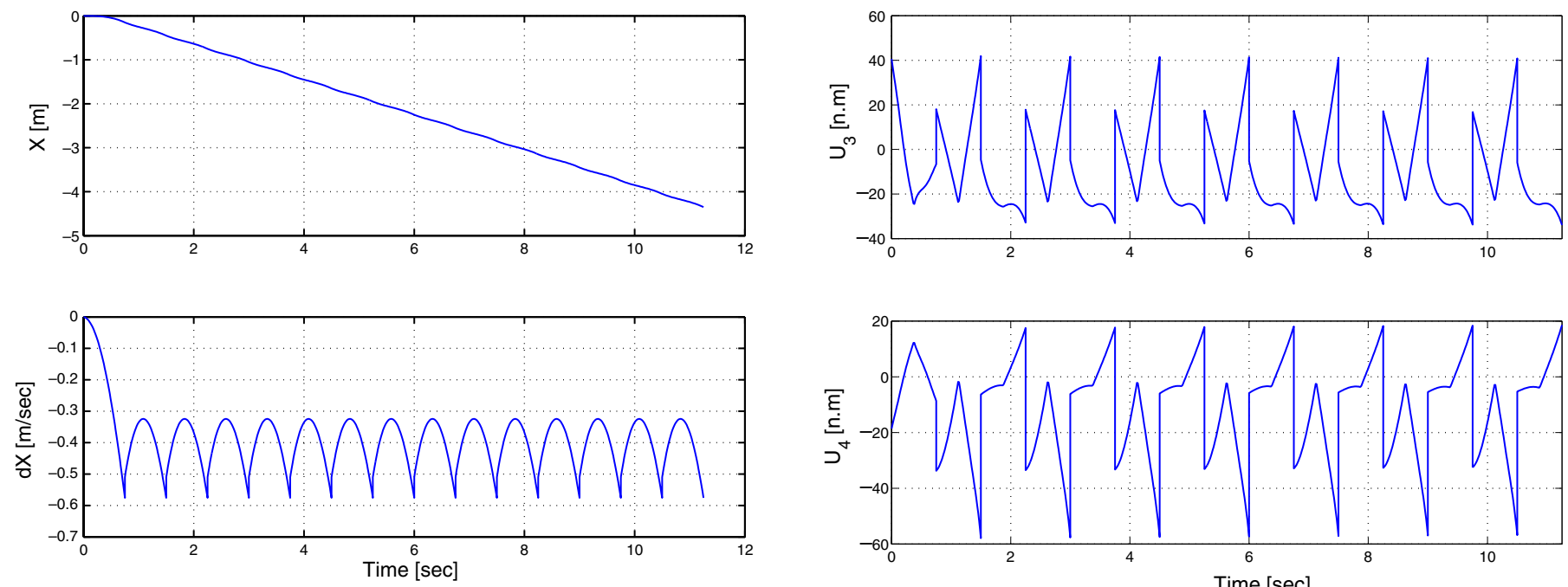

Fig. 17. (Sim 1) The $x$ position and velocity versus time.
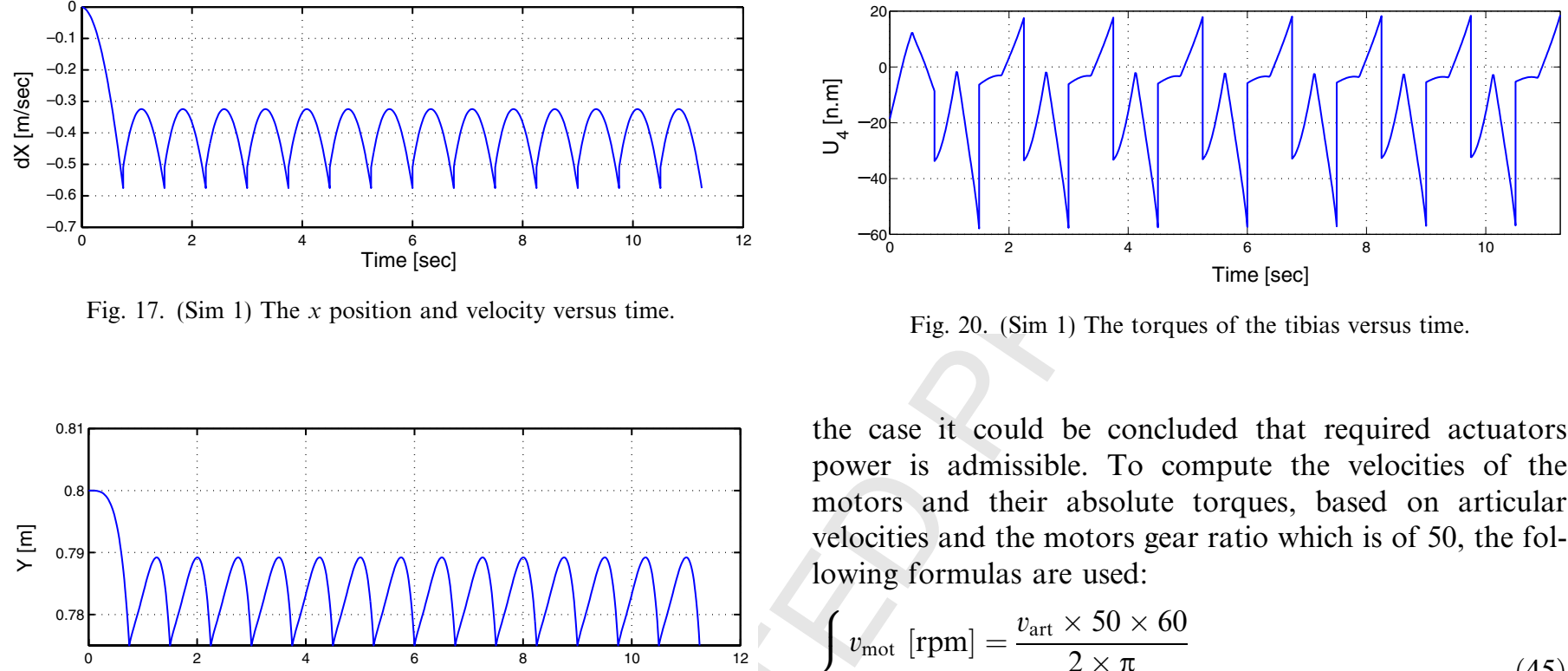

Fig. 20. (Sim 1) The torques of the tibias versus time.

the case it could be concluded that required actuators 774 power is admissible. To compute the velocities of the 775 motors and their absolute torques, based on articular 776 velocities and the motors gear ratio which is of 50, the fol- 777 lowing formulas are used:

$$
\left\{\begin{array}{l}
v_{\text {mot }}[\mathrm{rpm}]=\frac{v_{\text {art }} \times 50 \times 60}{2 \times \pi} \\
\tau_{\text {mot }}[\mathrm{N} \mathrm{m}]=\frac{\tau_{\text {art }}}{50}
\end{array}\right.
$$

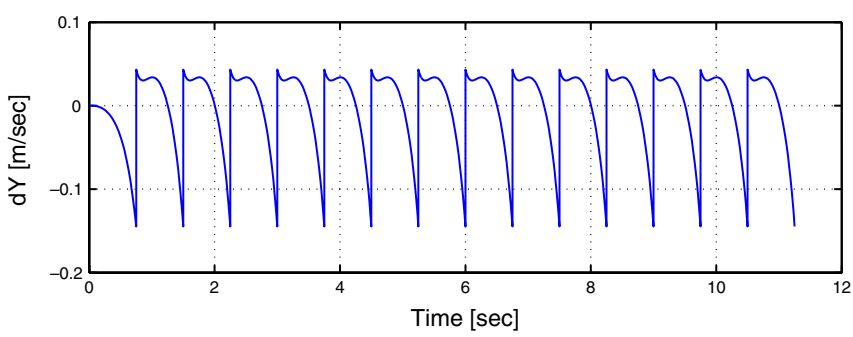

where $v_{\text {mot }}[\mathrm{rpm}]$ is the velocity of the motor shaft, $v_{\text {art }}[\mathrm{rad} /$ $\mathrm{s}]$ is the relative velocity between the two adjacent links of the concerned articulation, $\tau_{\mathrm{mot}}[\mathrm{N} \mathrm{m}]$ is the motor torque, and $\tau_{\text {art }}[\mathrm{N} \mathrm{m}]$ is the torque applied on the links.

The application of this verification technique is illustrated on Fig. 21, which depicts the shaft speed versus tor-

Fig. 18. (Sim 1) The $y$ position and velocity versus time.
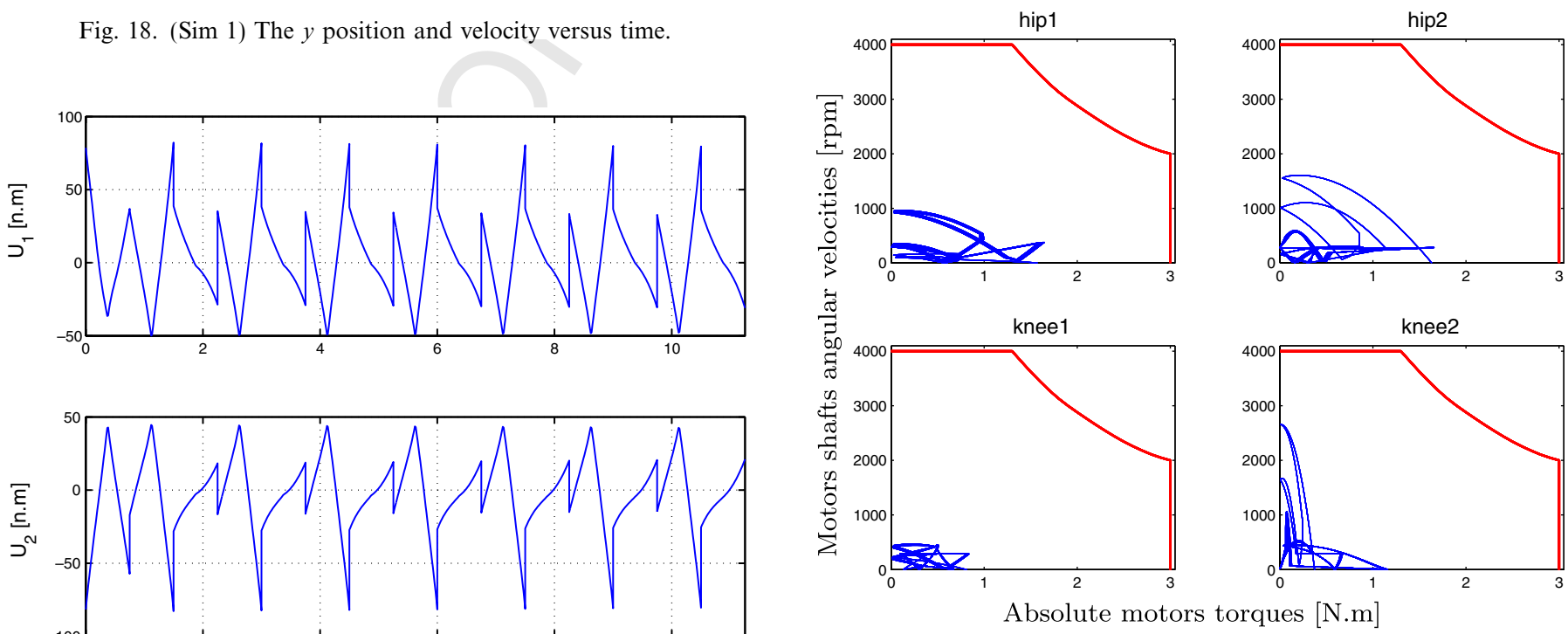

Fig. 21. (Sim 1) Absolute value of actuator angular velocities (revolutions per minute) versus absolute value of actuator torques $[\mathrm{N} \mathrm{m}]$, and admissible region. 
que, for the four robot actuators, where it could be clearly seen that the actuators power requirement is admissible. The contact foot interaction forces with ground are plotted versus time in Fig. 22, where we note that the condition of the friction Coulomb's law is largely satisfied (this is clearly seen through the amount of the ratio $\lambda_{t} / \lambda_{n}$ with respect to the friction coefficient which is $\mu_{0}=0.7$ ). Fig. 23 illustrates the movement of the robot by means of a set of walking stick figures (for the three first steps).

\subsubsection{Computation time evaluation}

In order to evaluate the computation time of the proposed control scheme, let us consider biped walking for 50 steps with a constant speed. The evaluation of the com0 puting time is displayed in Fig. 12, which represents the evolution of the computing time versus cycles (steps). The maximum value is given by $t_{\max }=0.97 \mathrm{~s}$. For real time
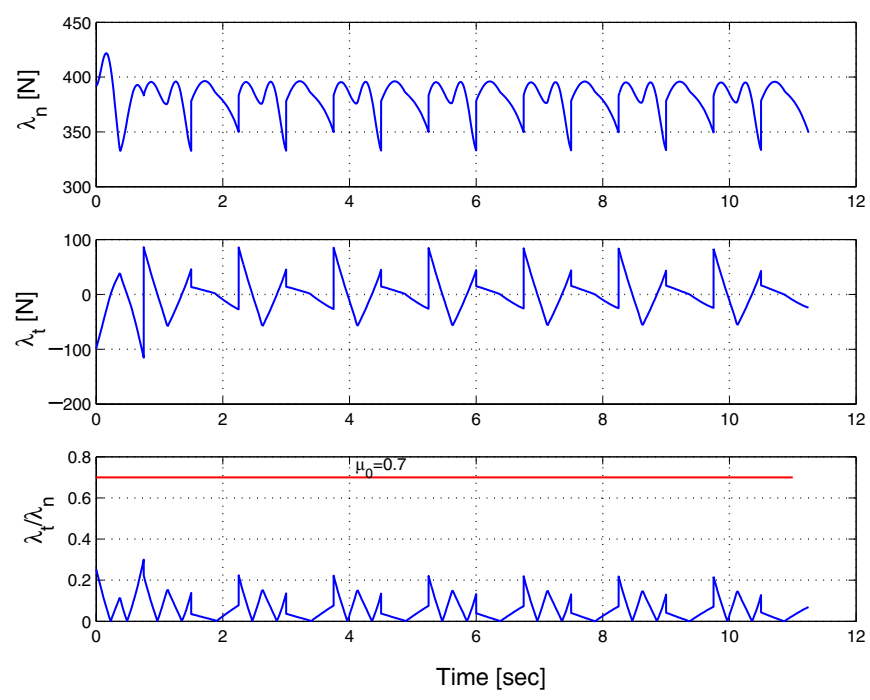

Fig. 22. (Sim 1) The stance foot interaction forces with ground and their ratio $\left(\lambda_{t} / \lambda_{n}\right)$.

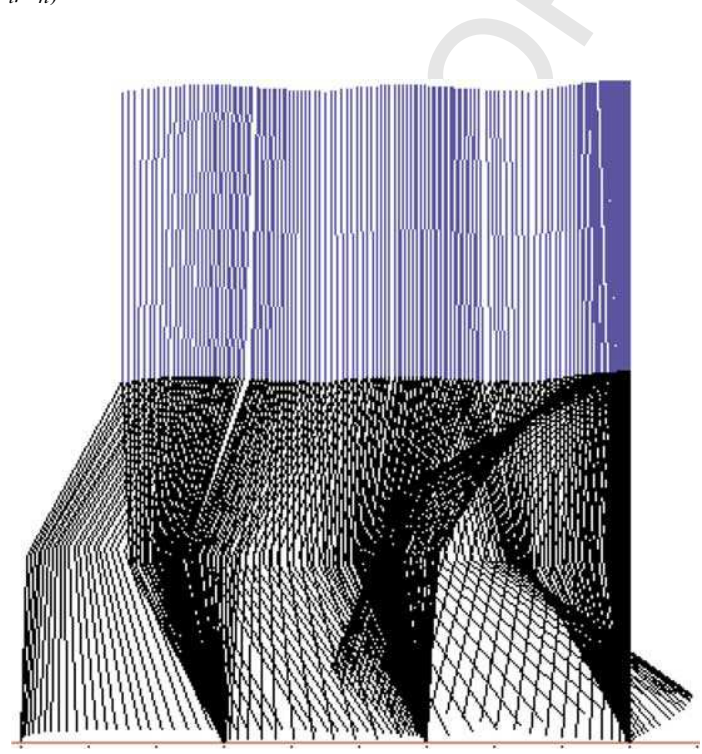

Fig. 23. (Sim 1) Stick figures of the walking robot. implementation the on-line optimization is replaced by an interpolation procedure. The basic idea is to define a grid on the space $\left(q_{1}, \dot{q}_{1}\right)$, and for all the points the optimization problem is resolved off-line to define the corresponding optimization parameter $p$, so that at the end of the procedure a look-up table is obtained. In the experiments this look-up table is used to find, for the chosen configuration, the optimization parameter at each sample time knowing the initial condition (position and velocity) on the unactuated coordinate.

\subsection{Simulation 2: transition between different mean walking velocities}

\section{3}

In this simulation, it is shown that the proposed feedback enables transitions between different mean walking velocities to be easily obtained. To show this, 46 walking cycles have been produced during which different desired velocities of $0.24 \mathrm{~m} / \mathrm{s}, 0.3 \mathrm{~m} / \mathrm{s}$ and $0.40 \mathrm{~m} / \mathrm{s}$ are successively applied during 12, 14 and 20 cycles respectively. Because of the proportional dependency between the duration of the cycle $t_{\mathrm{f}}$ and the mean walking speed we have chosen to change $t_{\mathrm{f}}$ under constant $d=0.3 \mathrm{~m}$ in order to increase (go faster), or to decrease (go slower) the walking speed. Since the step length is $0.3 \mathrm{~m}$, the choice of the cycle endtime corresponding to the yet mentioned speeds $(0.24$, 0.3 , and $0.40 \mathrm{~m} / \mathrm{s}$ respectively) is $(1.25,1$, and $0.75 \mathrm{~s} \mathrm{respec-}$ tively).

Fig. 24 shows the phase portrait of the unactuated coordinate (torso), where it is well shown the transition between the different stable limit cycles (each limit cycle is relative to a walking speed). In Fig. 25 the mean walking speed is plotted, showing thus the switching between the different proposed walking speeds. The behavior of the unactuated coordinate (torso) is illustrated in Fig. 26 giving its position as well as its velocity versus time. The cartesian coordinates (horizontal, respectively vertical) of the hips are depicted

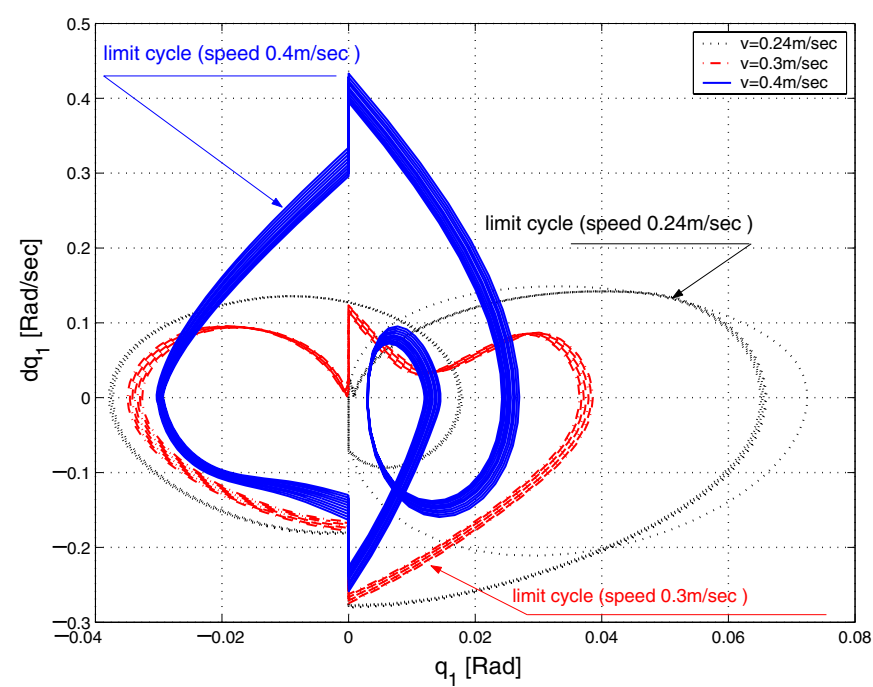

Fig. 24. (Sim 2) The phase portrait of the non actuated coordinate (torso). 


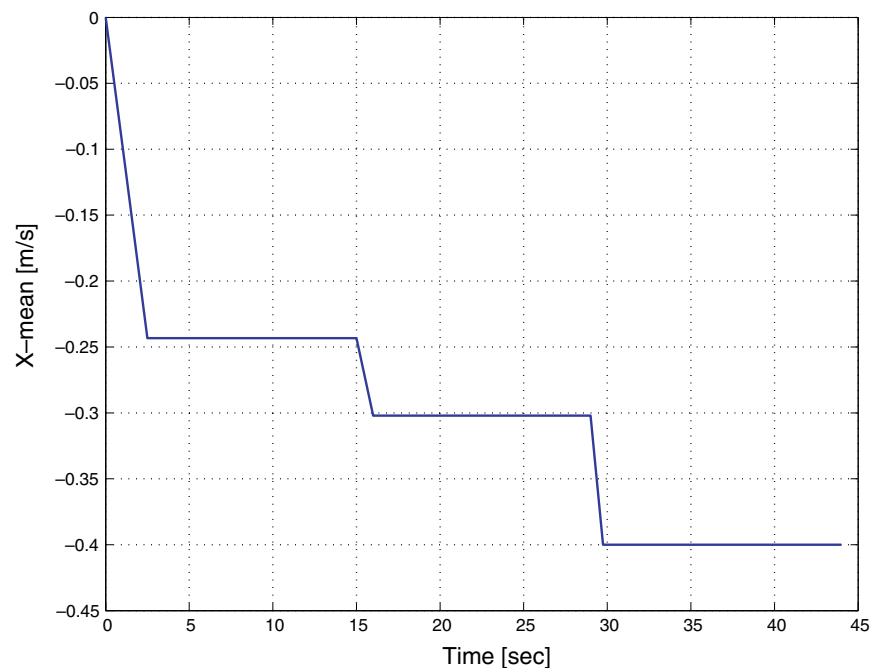

Fig. 25. (Sim 2) The mean walking velocity.
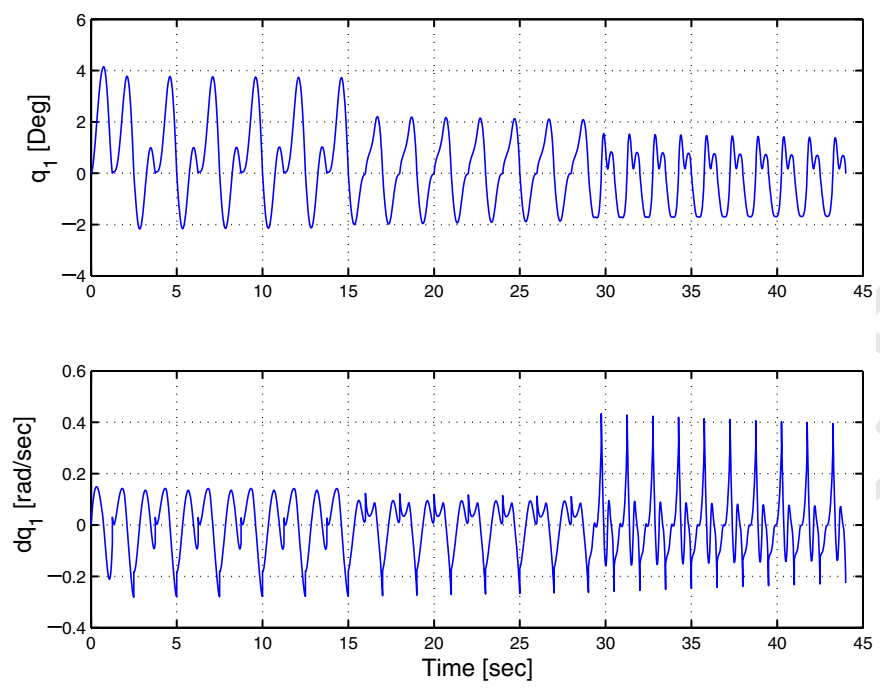

Fig. 26. (Sim 2) The torso position and velocity versus time.

838 respectively in Figs. 28 and 29, and a more illustrative plot 839 of the hip's trajectory in the sagittal plane is given in 840 Fig. 27.

841 The generated torques are plotted in Figs. 32 and 33 for 842 the femurs and tibias respectively, while in Fig. 30 it is 843 checked that power requirement remain within the permit844 ted limit. The contact forces with ground of the stance leg 845 foot are depicted in Fig. 31, where we see clearly that the 846 robot keeps contact with ground during walking.

847 5.4. Simulation 3: robustness against parameters

848 uncertainty

849 In order to investigate the robustness of the proposed 850 controller, let us introduce parameter uncertainties. The 851 inertias of the robot links, namely $I_{1}$ (the torso), $I_{3}$ (the 852 femur) and $I_{4}$ (the tibia) cf. Table 1 , are considered with 853 an uncertainty of $10 \%$ of the their nominal values, that is

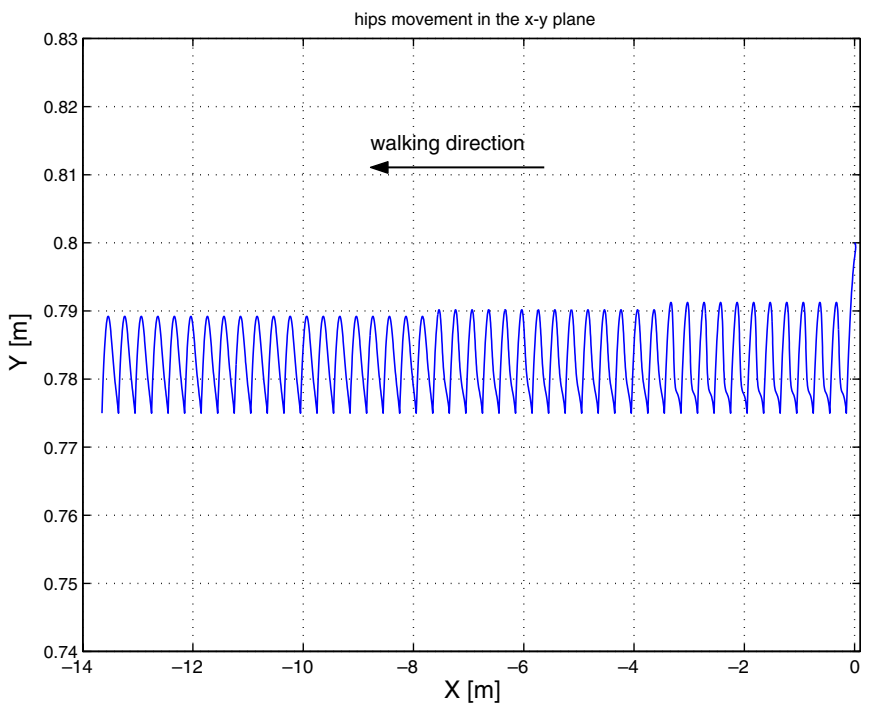

Fig. 27. (Sim 2) The hips movement trajectory.
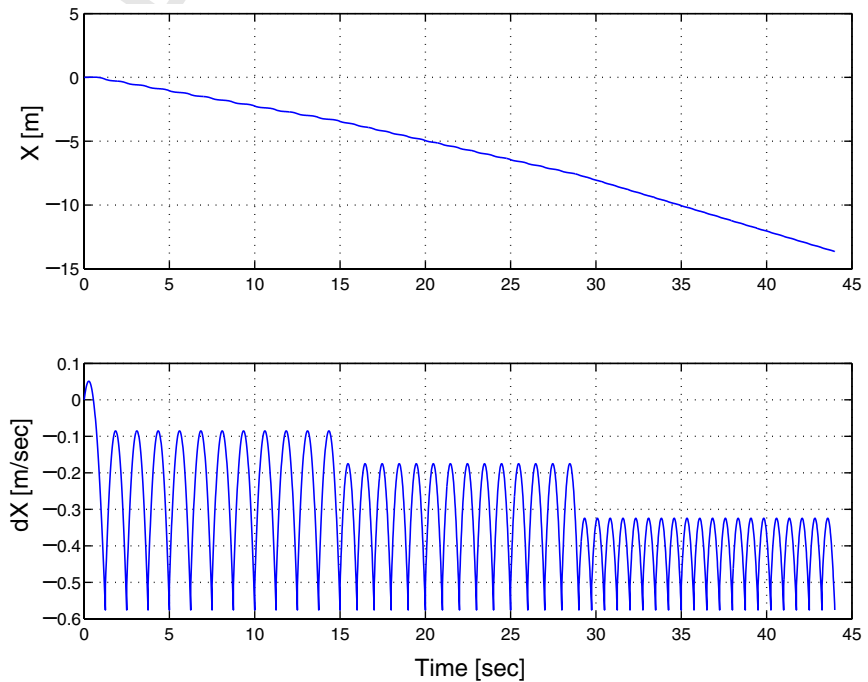

Fig. 28. (Sim 2) The $x$ position and velocity versus time.
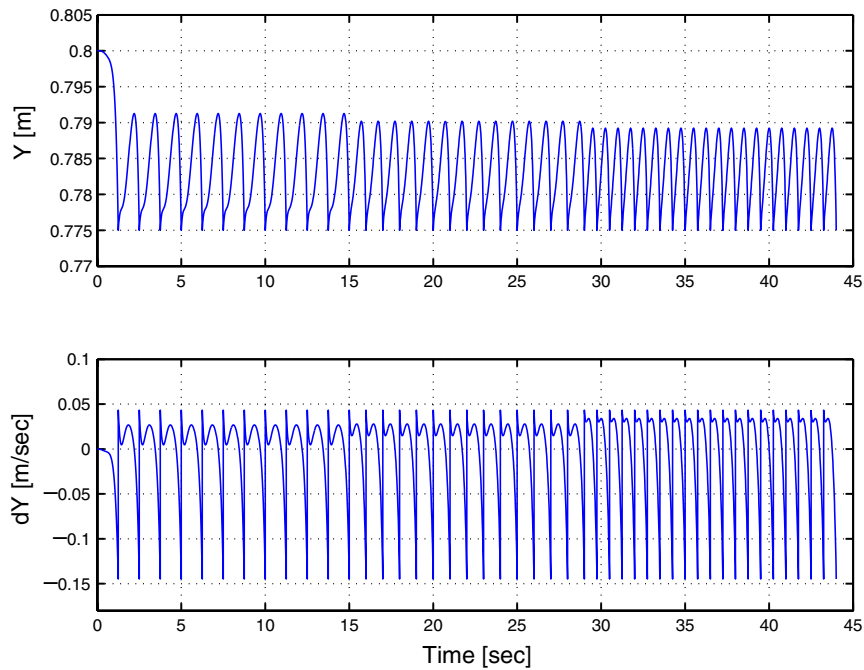

Fig. 29. (Sim 2) The $y$ position and velocity versus time. 


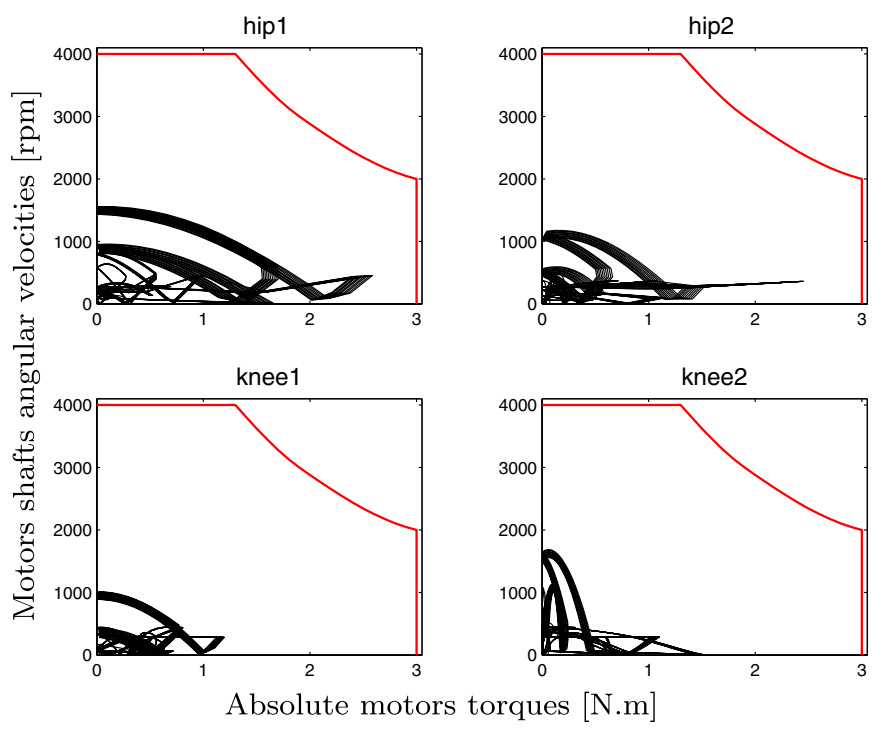

Fig. 30. (Sim 2) Absolute value of actuator angular velocities (revolutions per minute) versus absolute value of actuator torques $[\mathrm{N} \mathrm{m}]$, and admissible region.
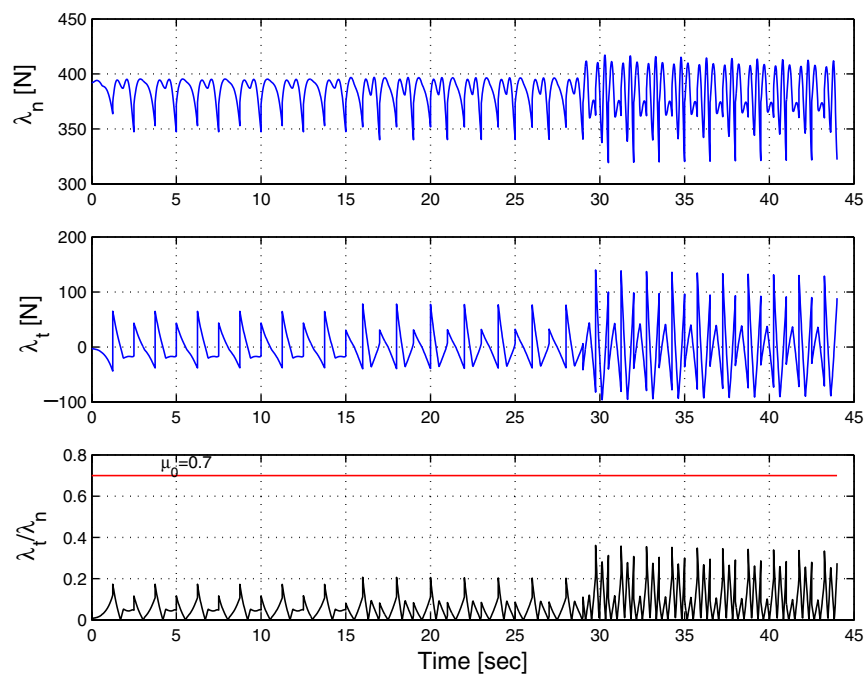

Fig. 31. (Sim 2) The stance foot interaction forces with ground and their ratio $\lambda_{t} / \lambda_{n}$

$I_{1 u}=I_{1}+\Delta I_{1} ; \quad I_{3 u}=I_{3}+\Delta I_{3} ; \quad I_{4 u}=I_{4}+\Delta I_{4}$

where the uncertainties $\Delta I_{i}=0.1 I_{i}$, for $i \in\{1,3,4\}$. Figs. 34-39 present the corresponding simulation results over 12 walking steps.

In Fig. 34 the positions and velocities of the first leg femur are plotted for the nominal system (solid line), as well as for the uncertain system (dashed line). Whereas in Fig. 35, the positions and velocities of the tibia are plotted. It can be seen clearly that the introduced uncertainty affects more the femur coordinates.

The behavior of the unactuated coordinate (torso) is represented in Fig. 36 which plots the evolution of its position and velocity versus time. A convergence to a new stable cyclic trajectory is observed. This fact can be seen also

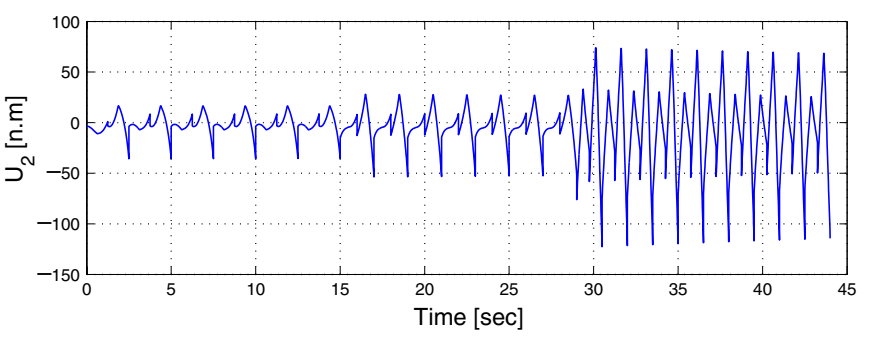

Fig. 32. (Sim 2) The torques of the femurs versus time.
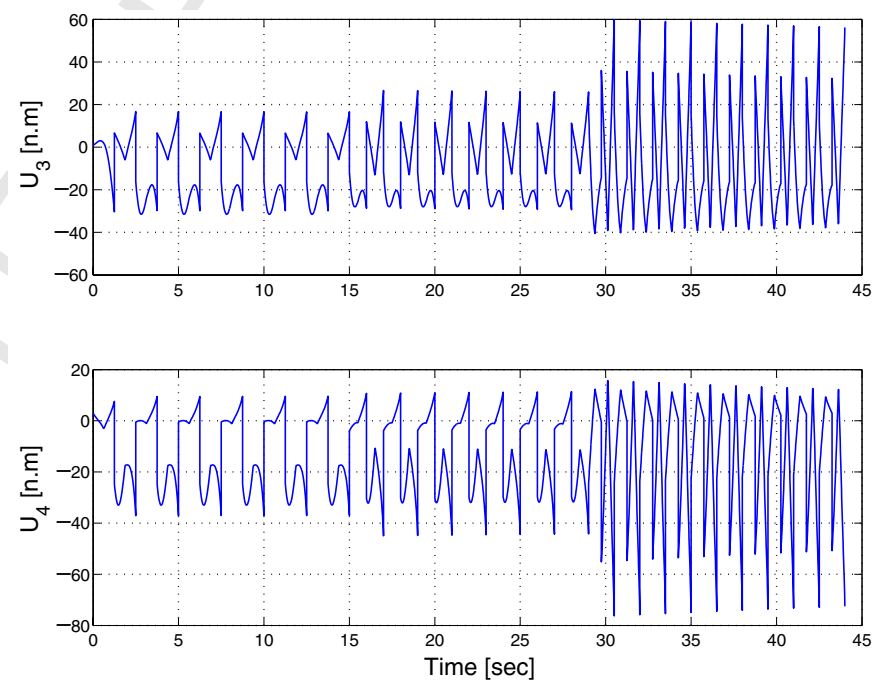

Fig. 33. (Sim 2) The torques of the tibias versus time.

on the phase portrait of Fig. 37, where a convergence to a neighborhood of a new limit cycle (different from that of the nominal system) of length 2 is observed for the uncertain system.

In Figs. 38 and 39 the control inputs of the robot are plotted, they represent the torques generated by the proposed controller for the femurs articulations (Fig. 38) and for the tibias articulations (Fig. 39). For both figures the uncertain system torques are slightly different from those of the nominal system.

Let us now consider an other test of the robustness of the proposed control approach. This time consider an uncertainty of $15 \%$ on the mass of the unactuated coordinate (torso), that is

$m_{1 u}=m_{1}+\Delta m_{1} ; \quad \Delta m_{1}=15 \%$

To see the effect of the introduced uncertainty on the 884

closed-loop system two figures are given. On Fig. 40 the 

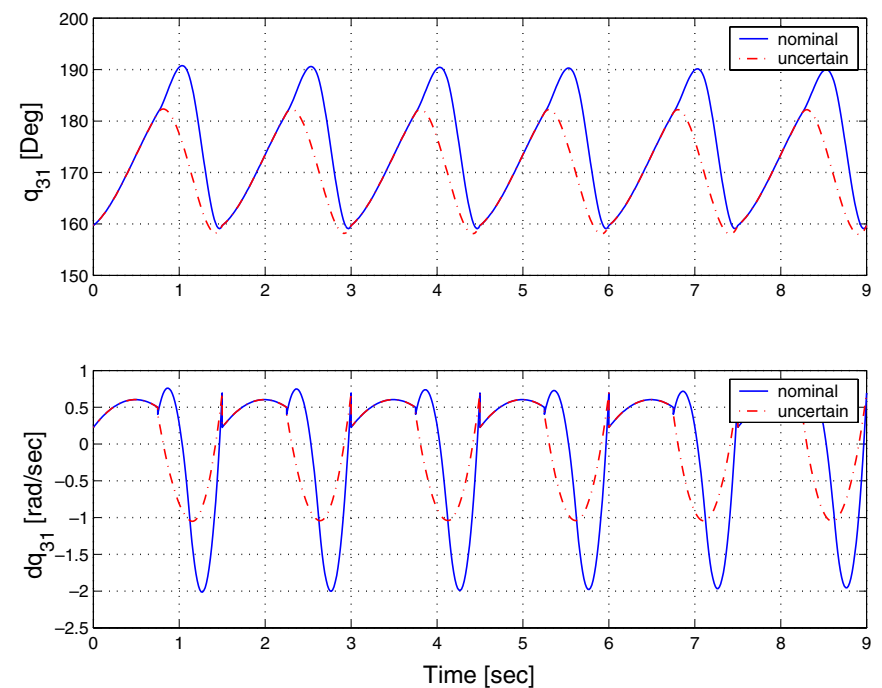

Fig. 34. (Sim 3) Position and velocity of the femur of the first leg.
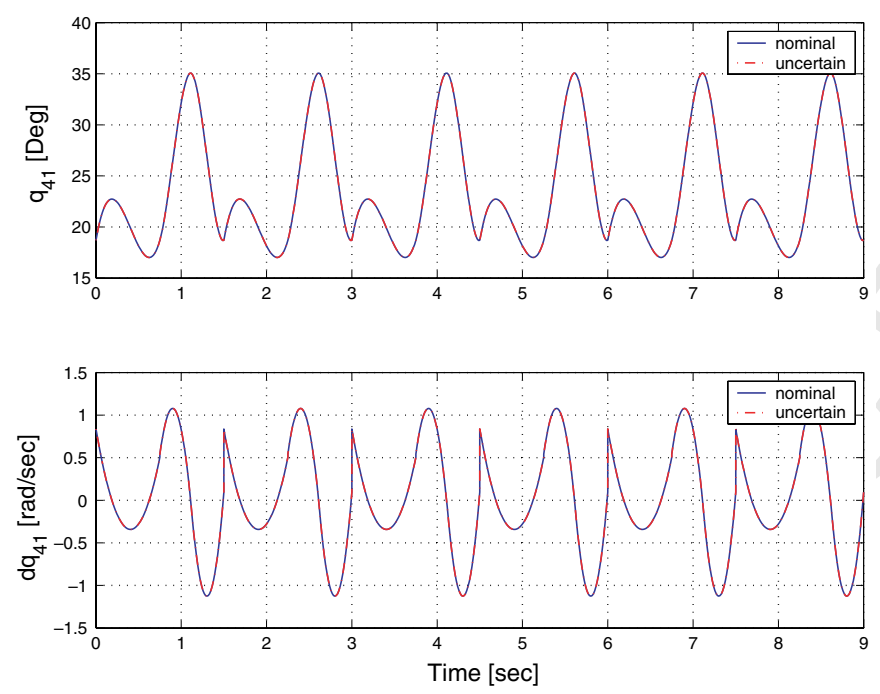

Fig. 35. (Sim 3) Position and velocity of the tibia of the first leg.
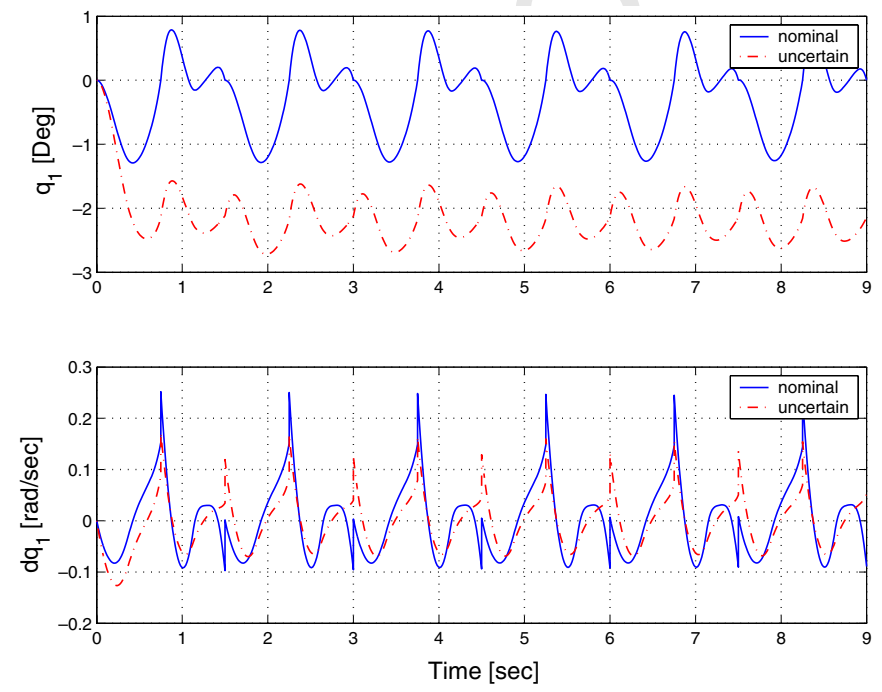

Fig. 36. (Sim 3) Position and velocity of the torso.

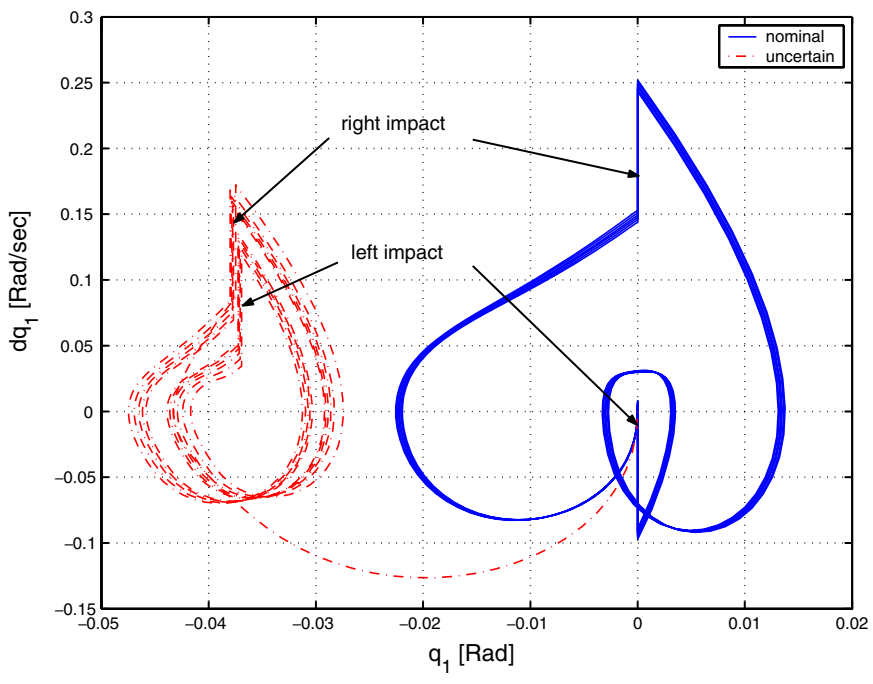

Fig. 37. (Sim 3) Phase portrait of the torso.
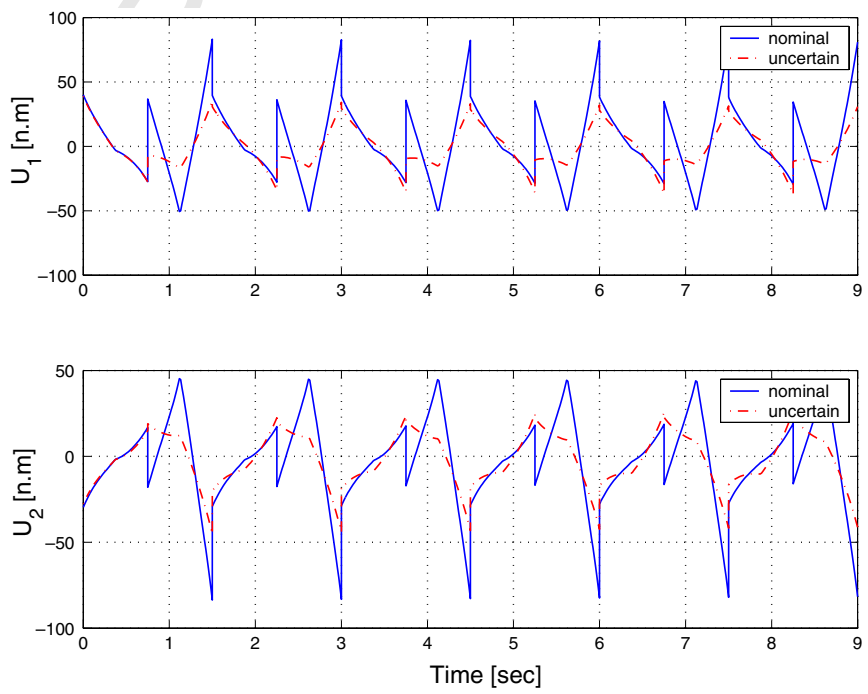

Fig. 38. (Sim 3) Torques of the femurs.
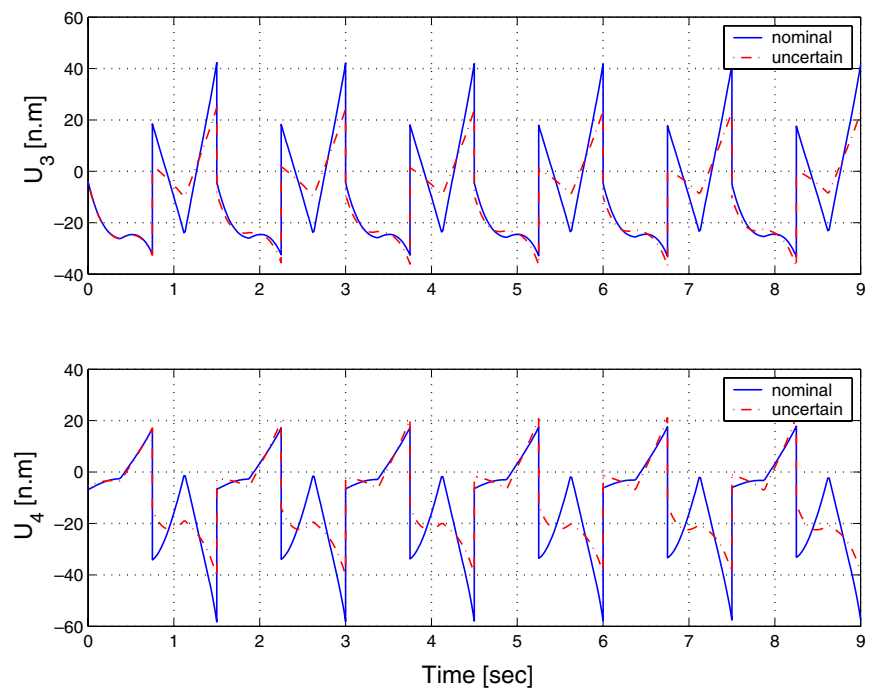

Fig. 39. (Sim 3) Torques of the tibias. 

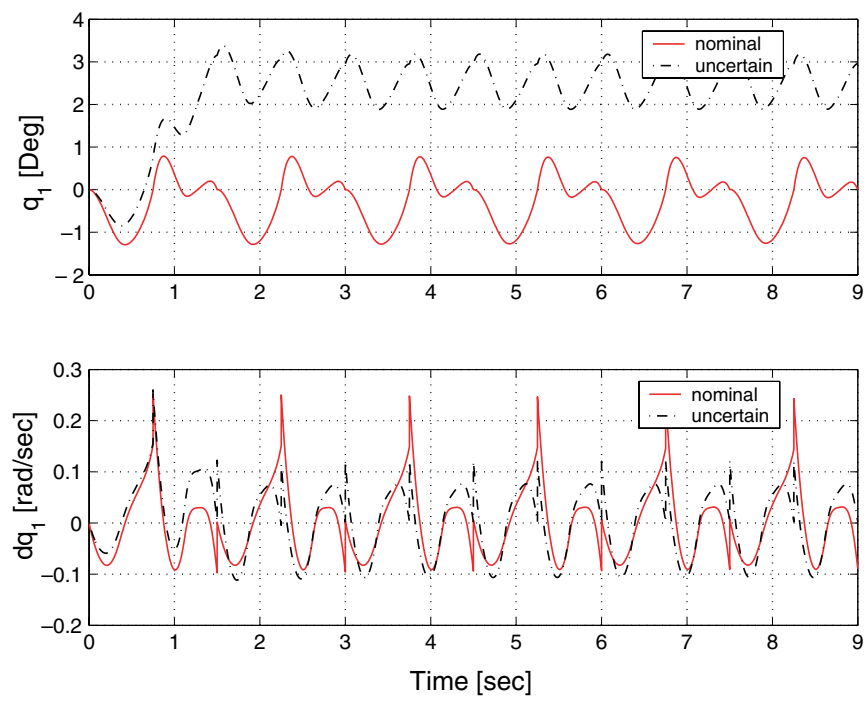

Fig. 40. (Sim 3) Position and velocity of the torso.

evolution of the position and the velocity of the torso are displayed. Where it could be seen clearly the convergence to a new cyclic trajectory for the uncertain system. An other interesting point result in the periodicity of the velocity trajectory which is of 1 cycle. This last fact could be better observed on Fig. 41 of the phase portrait, where one notices a convergence to an other stable limit cycle. However the new limit cycle is of length 1 , consequently the introduced uncertainty on the torso masse has induced a deformation of the limit cycle.

\subsection{Simulation 4: robustness against ground irregularities}

The aim is to investigate the robustness of the proposed controller, against ground irregularities. Let us make the robot walking on a horizontal surface with a stair at a distance of $1.4 \mathrm{~m}$ from the robot, the stair is $1 \mathrm{~cm}$ height.

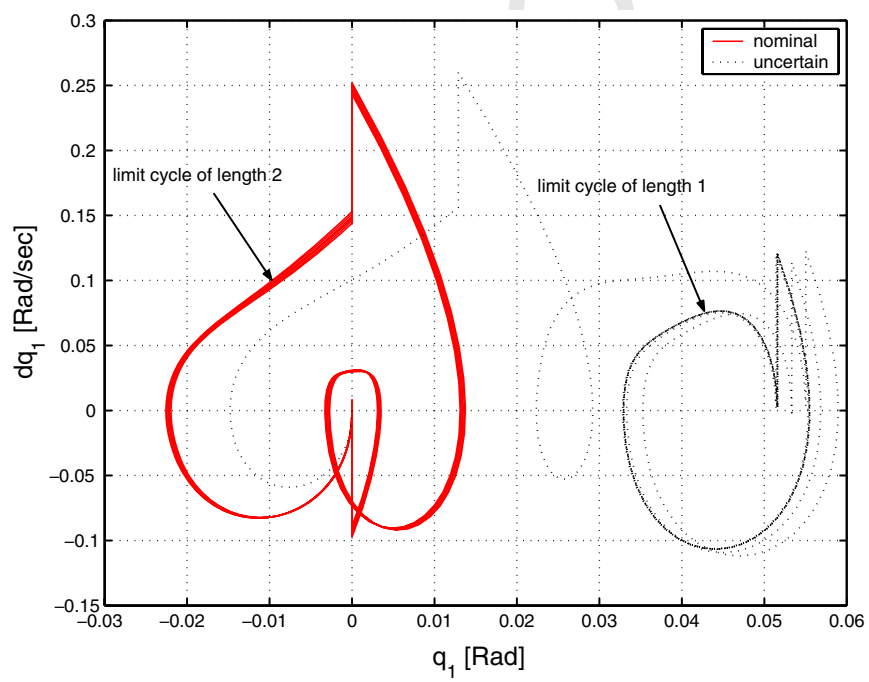

Fig. 41. (Sim 3) Phase portrait of the torso.
According to the robot configuration, namely the step 902 length $d=0.3 \mathrm{~m}$, the robot hits the stair during the fifth walking step.

The approach parameters are the same as previous simulations except the weighting matrix in the optimization criterion which is chosen $Q=\operatorname{Diag}\{1,0.1\}$, and the step duration which is of $t_{\mathrm{f}}=1 \mathrm{~s}$ now. This change in these two parameters have been adopted because it gives a better results, namely a configuration with these parameters choice is more robust than the configuration with the old values.

The corresponding simulation results are depicted in Figs. 42-47. It is worth to note that the impact is detected at the instants $t=1 \mathrm{~s}$ for all steps, except for the step during which it hits the stair (fifth step), where the impact instant corresponding to this last one is $t=0.935 \mathrm{~s}$, which is before the expected time $t=1 \mathrm{~s}$.
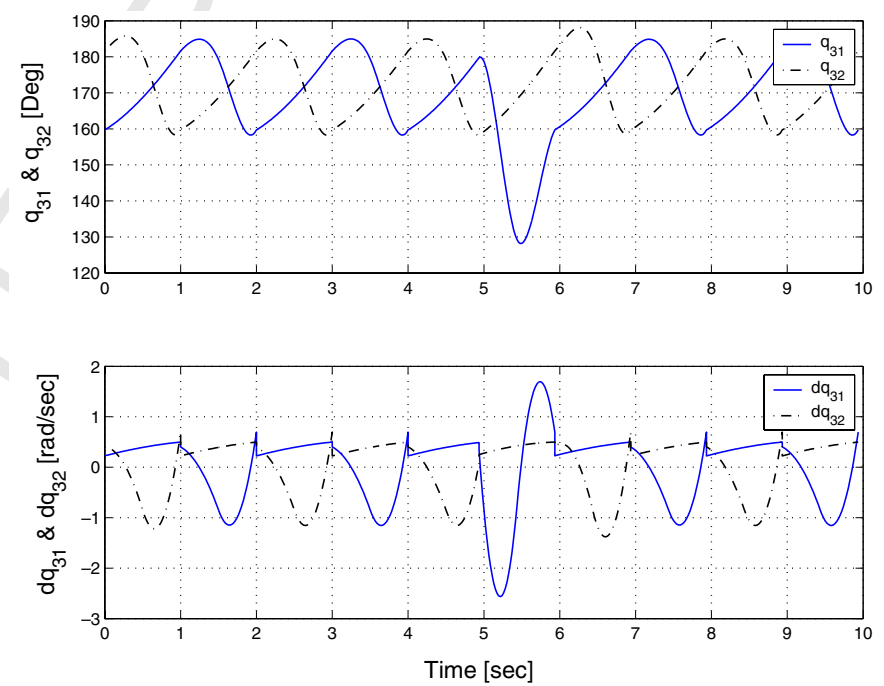

Fig. 42. (Sim 4) Position and velocity of the robot femurs.
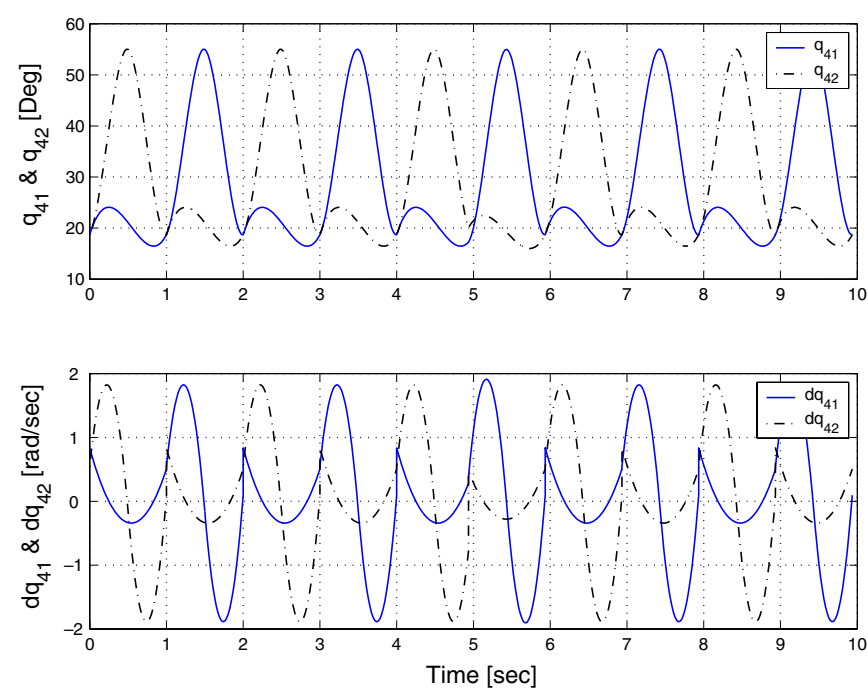

Fig. 43. (Sim 4) Position and velocity of the robot tibias. 

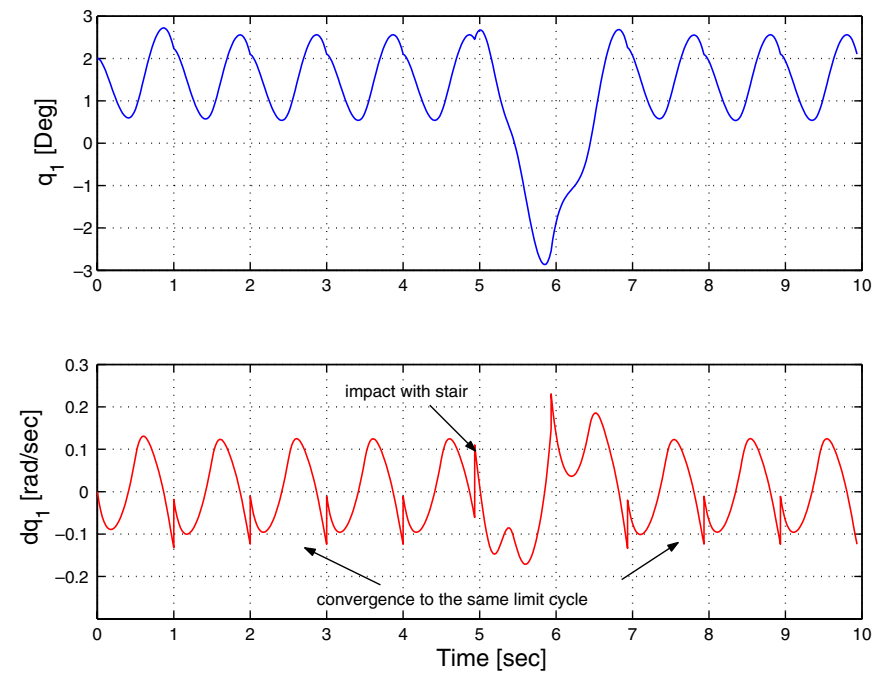

Fig. 44. (Sim 4) Position and velocity of the torso.

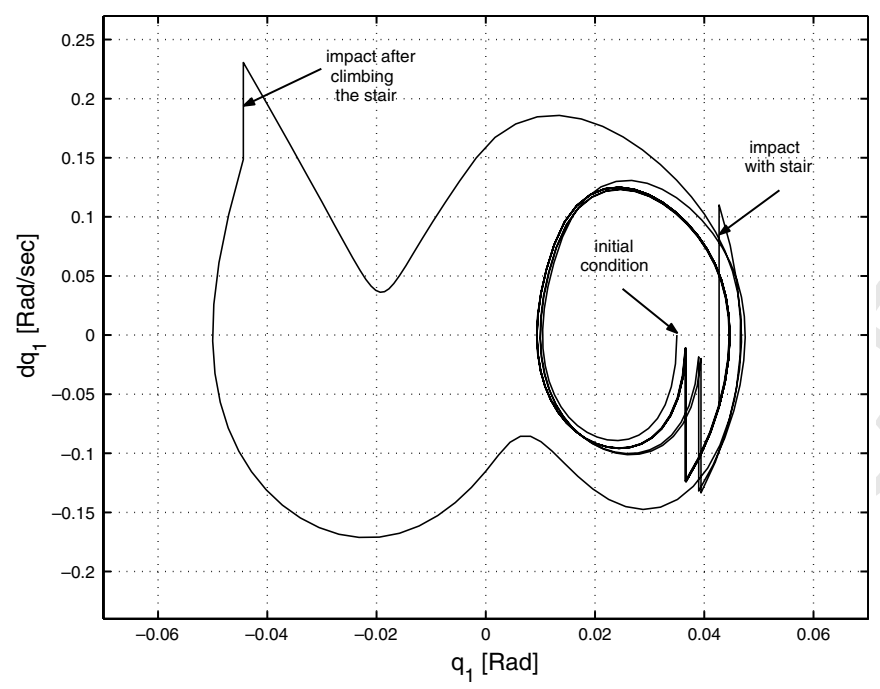

Fig. 45. (Sim 4) The phase portrait of the torso.

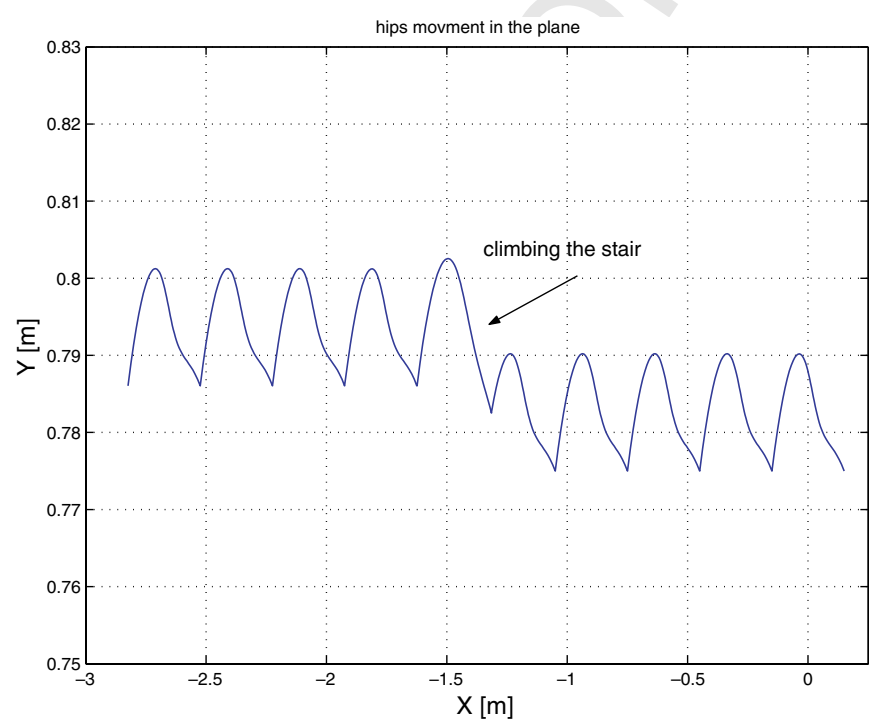

Fig. 46. (Sim 4) The hips trajectory in the sagittal plane.

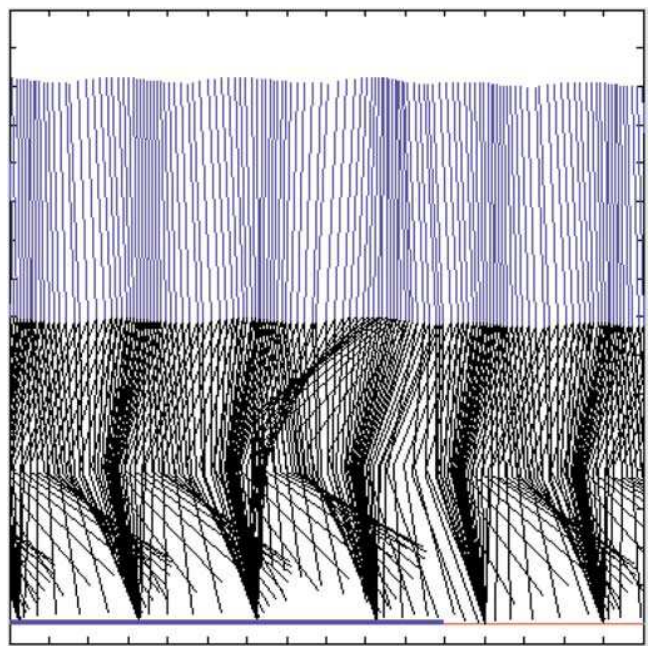

Fig. 47. (Sim 4) Stick figures of stair climbing.

Figs. 42 and 43 display the positions and velocities of the 919 actuated robot's limbs versus time. The effect of the unex- 920 pected impact could be seen on Fig. 42 as a removal from 921 the cyclic trajectory followed by a convergence to the same 922 trajectory. This effect can also be observed on the behavior 923 of the unactuated coordinate (torso). Especially Fig. 44924 which shows the evolution of the angular position and 925 velocity of the torso, and Fig. 45 which displays its phase 926 portrait. On both figures a removal from the cyclic trajec- 927 tory is observed, followed by a convergence to the same 928 trajectory. It is worth to note that the limit cycle is of 929 length 1 for this simulation. Fig. 46 depicts the hips trajec- 930 tory in the sagittal plane, whereas Fig. 47 illustrates 931 through a stick figures of the robot postures the climbing 932 of the stair.

The limit of stair height beyond it the robot falls is closely related to the chosen configuration. For the actual configuration the limit is of $5 \mathrm{~cm}$. Nevertheless this limit could be increased by changing the configuration, or the approach parameters.

\section{Conclusion and future work}

In this paper, a nonlinear low dimensional predictive control approach is proposed for the control of RABBIT, a walking five-link, seven d.o.f. under-actuated biped robot. The basic idea of the approach is to split up the vector of coordinates into actuated and unactuated variables. Then on-line optimization is used to update reference trajectories on the actuated coordinates, to aim to enhance the behavior as well as the stability of the unactuated variables.

The stability analysis of the resulting closed-loop system is carried out using a graphical tool based on the Poincaré section. Sufficient conditions for the stability of the motion are proposed and a concrete computation procedure is given to estimate the corresponding region of attraction related to the zero-dynamics of the closed-loop system. The particular case of scalar predictive control is success-
940 941 942 943 944 945 946 947 948 949 950 951 952 953 954 
955 fully investigated by simulation and a reasonable regions of 956 attraction are obtained.

957 The resulting feedback seems to be real-time implement958 able thanks to the low dimension of the optimization 959 problem.

960 The whole framework is illustrated through simulation 961 case studies. Indeed four simulations are proposed. In the 962 first one walking at constant average speed starting from 963 rest is investigated, while the second scenario concerns 964 switching between different walking speeds. Robustness 965 of the proposed nonlinear predictive based-upon controller 966 is verified through the two last applications. In the first one 967 a robot model including parameters uncertainties (namely 968 uncertainties on inertias and masses) is considered, while 969 in the second, ground irregularities are considered. In spite 970 of these both significant disturbances the controller is able 971 to guide the robot suitably for walking while keeping it sta972 ble (no slipping, no falling).

973 In simulations, after each impact the reference trajecto974 ries on actuated coordinates are computed based on a pre975 defined step frequency. These trajectories are tracked while 976 checking at each decision instant if there is impact (RABBIT 977 prototype is equipped with switches at feet used to detect 978 impacts). If an impact is detected, the two legs are re979 labelled, the configuration of the robot is measured and a 980 new step starts up.

981 Future works may include other features, that should be 982 deeply investigated. In particular, one may be able to 983 choose the design parameters $t_{f}, z_{2}^{\mathrm{f}}$ and $Q$ in order to opti984 mize some desired feature (mean energy, torque, robust985 ness). However, the key future work is naturally the 986 experimentations. This is currently in progress.

\section{References} Canudas de Wit C, et al. Rabbit: a testbed for advanced control theory. IEEE Contr Syst Mag 2003;23(5):57-79.
[11] Fontes FACC. A general framework to design stabilizing nonlinear model predictive controllers. Syst Control Lett 2001;42(2):127-43.

[12] Grizzle JW, Abba G, Plestan F. Asymptotically stable walking for biped robots: analysis via systems with impulse effects. IEEE Trans Automat Contr 2001;46(1):51-64.

[13] Gubina F, Hemami H, McGee RB. On the dynamic stability of biped locomotion. IEEE Trans Biomed Eng 1974;21(12):102-8.

[14] Hardt M, Kreutz-Delgado K, Helton J. Minimal energy control of a biped robot with numerical methods and a recursive symbolic dynamic model. In: Proceedings of 37th IEEE conference on decision contr., Florida, USA, 1998. p. 413-16.

[15] Hurmuzlu Y, Marghitu DB. Rigid body collisions of planar kinematic chains with multiple contact points. Int $\mathbf{J}$ Rob Res 1994;13(1):82-92.

[16] Katoh R, Mori M. Control method of biped locomotion giving asymptotic stability of trajectory. Automatica 1984;20(4):405-14.

[17] Khalil H. Nonlinear systems. Second Edition. Upper Saddle River: Prentice Hall; 1996.

[18] Kun A, Miller W. Adaptive dynamic balance of an experimental biped robot. In: Proceedings of IEEE conference on robotics automatics, 1996.

[19] Magdalena L, Monasterio-Huelin F. A fuzzy logic controller with learning through the evolution of its knowledge base. Int JApprox Reason 1997;16(3/4):335-58.

[20] Mayne DQ, Rawlings JB, Rao CV, Scokaert PO. Constrained model predictive control: stability and optimality. Automatica 2000;36:789-814.

[21] McGeer T. Passive dynamic walking. Int $\mathbf{J}$ Robot Res 1990;9(2):62-82.

[22] Miura H, Shimoyama I. Dynamic walk of a biped. Int J Rob Res 1984;3(2):60-74.

[23] Mrecki A, Waldron K. Human and machine locomotion. LNCIS. Udine, Italy: Springer Verlag; 1997.

[24] Nicholls E. Bipedal dynamic walking in robotics. Honours thesis, University of western Australia, 1998.

[25] Park JH. Impedance control for biped robot locomotion. IEEE Trans Robot Automat 2001;17(6):870-82.

[26] Plestan F. Commande de la marche d'un bipède type rabbit avec trajectoires optimales et évaluation de la robustesse. In: CIFA 2002, 2002. p. 516-21.

[27] Plestan F, Grizzle JW, Westervelt ER, Abba G. Stable walking of a 7 dof biped robot. IEEE Trans Robot Automat 2003;19(4):653-68.

[28] Pratt J, Pratt G. Intuitive control of a planar bipedal walking robot. In: Proceedings of IEEE Conference on Robotics Automat. Leuven, Belgium, 1998. p. 2014-21.

[29] Raibert MH. Legged robots. Commmun ACM 1986;29:499-514.

[30] Rostami M, Bessonnet G. Impactless sagittal gait of a biped robot during the single support phase. In: Proceedings of the IEEE Conference on Robotics Automat. Leuven, Belgium, 1998. p. 138591.

[31] Roussel L, Canudas C, Goswami A. Generation of energy optimal complete gait cycles for biped robots. In: Proceedings of the IEEE Conference on Robotics Automat. Leuven, Belgium, 1998. p. 203641

[32] Sciavicco L, Siciliano B. Modeling and control of robot manipulators. New York: McGraw Hill; 1996.

[33] Spong M. Passivity based control of the compass gait biped. In: IFAC World Congress, Beijing, China, 1999.

[34] Westervelt ER. Towards a coherent framework for the control of planar biped locomotion. PhD thesis, University of Michigan, 2003.

[35] Westervelt ER, Grizzle JW, Canudas de Wit C. Switching and pi control of walking motions of planar biped walkers. IEEE Trans Automat Contr 2003;48(2):308-12.

[36] Westervelt ER, Grizzle JW, Koditschek DE. Hybrid zero dynamics of planar biped walkers. IEEE Trans Automat Contr 2003;48(1):42-56.
1012

1013

1014

1015

1016

1017

1018

1019

1020

1021

1022

1023

1024

1025

1026

1027

1028

1029

1030

1031

1032

1033

1034

1035

1036

1037

1038

1039

1040

1041

1042

1043

1044

1045

1046

1047

1048

1049

1050

1051

1052

1053

1054

1055

1056

1057

1058

1059

1060

1061

1062

1063

1064

1065

1066

1067

1068

1069

1070

1071

1072

1073

1074

1075

1076

1077 